\title{
MODULAR COMPLEXITY ANALYSIS FOR TERM REWRITING*
}

\author{
HARALD ZANKL $^{a}$ AND MARTIN KORP ${ }^{b}$ \\ ${ }^{a, b}$ Institute of Computer Science, University of Innsbruck, Austria \\ e-mail address: \{harald.zankl,martin.korp\}@uibk.ac.at
}

\begin{abstract}
All current investigations to analyze the derivational complexity of term rewrite systems are based on a single termination method, possibly preceded by transformations. However, the exclusive use of direct criteria is problematic due to their restricted power. To overcome this limitation the article introduces a modular framework which allows to infer (polynomial) upper bounds on the complexity of term rewrite systems by combining different criteria. Since the fundamental idea is based on relative rewriting, we study how matrix interpretations and match-bounds can be used and extended to measure complexity for relative rewriting, respectively. The modular framework is proved strictly more powerful than the conventional setting. Furthermore, the results have been implemented and experiments show significant gains in power.
\end{abstract}

\section{INTRODUCTION}

Term rewriting is a Turing complete model of computation. As an immediate consequence all interesting properties are undecidable. Nevertheless many powerful techniques have been developed to establish termination. The majority of these techniques have been automated successfully. This development has been stimulated by the international competition of termination tools 1 Most automated analyzers gain their power from a modular treatment of rewrite systems (typically via the dependency pair framework [2, 10, 25]).

For terminating rewrite systems Hofbauer and Lautemann [13] consider the length of derivations as a measurement for the complexity of rewrite systems. The resulting notion of derivational complexity relates the length of a rewrite sequence to the size of its starting term. Thereby it is, e.g., a suitable metric for the complexity of deciding the word problem for a given confluent and terminating rewrite system (since the decision procedure rewrites terms to normal form). If one regards a rewrite system as a program and wants to estimate the maximal number of computation steps needed to evaluate an expression to a result, then the special shape of the starting terms - a function applied to data which is in normal

2012 ACM CCS: [Theory of computation]: Design and analysis of algorithms; Computational complexity and cryptography - Complexity theory and logic; Logic; Formal languages and automata theory.

Key words and phrases: term rewriting, complexity analysis, relative complexity, derivation height.

* A preliminary version of this article appeared in RTA 2010.

${ }^{a}$ This research is supported by FWF (Austrian Science Fund) project P18763.

1 http://termcomp.uibk.ac.at 
form - can be taken into account. Hirokawa and Moser [11] identified this special form of complexity and named it runtime complexity.

To show (feasible) upper complexity bounds currently few techniques are known. Typically termination criteria are restricted such that complexity bounds can be inferred. The early work by Hofbauer and Lautemann [13] considers polynomial interpretations, suitably restricted, to admit quadratic derivational complexity. Match-bounds [8] and arctic matrix interpretations [16] induce linear upper bounds on the derivational complexity and triangular matrix interpretations 20 admit at most polynomially long derivations (the dimension of the matrices yields the degree of the polynomial) in the size of the starting term. All these methods share the property that until now they have been used directly only, meaning that a single termination technique has to orient all rules in one go. However, using direct criteria exclusively is problematic due to their restricted power.

In [11,12] Hirokawa and Moser lifted many aspects of the dependency pair framework from termination analysis into the complexity setting, resulting in the notion of weak dependency pairs. So for the special case of runtime complexity for the first time a modular approach has been introduced. There the modular aspect amounts to using different interpretation based criteria for (parts of the) weak dependency graph and the usable rules. However, still all rewrite rules considered must be oriented strictly in one go and only restrictive criteria may be applied for the usable rules. A further drawback of weak dependency pairs is that they may only be used for bounding runtime complexity while there seems to be no hope to generalize the method to derivational complexity.

In this article we present a different approach which admits a fully modular treatment. The approach is general enough that it applies to derivational complexity (and hence also to runtime complexity) and basic enough that it allows to combine completely different complexity criteria such as match-bounds and (triangular) matrix interpretations. By the modular combination of different base methods also gains in power are achieved. These gains come in two flavors. On the one hand our approach allows to obtain lower complexity bounds for several rewrite systems where bounds have already been established before and on the other hand we found bounds for systems that could not be dealt with so far automatically. More specifically, there are systems where the modular combination of different criteria allows to establish an upper bound while any of the involved methods cannot succeed on its own.

The remainder of the article is organized as follows. In Section 2 preliminaries about term rewriting and complexity analysis are fixed. Afterwards, Section 3 familiarizes the reader with the concept of a suitable complexity measurement for relative rewriting. Furthermore, it formulates a modular framework for complexity analysis based on relative complexity. Criteria for measuring relative complexity via interpretations and match-bounds are presented in Sections 4 and 5, respectively. In Section [6 we show that the modular setting is strictly more powerful than the conventional approach. Our results have been implemented in the complexity prover GT. The technical details can be inferred from Section 7 . Section 8 is devoted to demonstrate the power of the modular treatment by means of an empirical evaluation. Section 9 concludes.

This article is a restructured and extended version of [31. It also incorporates the results from the two notes [30,32] presented at informal workshops. Furthermore results and presentation have been generalized to address both derivational and runtime complexity. 


\section{Preliminaries}

We assume familiarity with (relative) term rewriting [4,9,24, Let $\mathcal{F}$ be a signature and $\mathcal{V}$ a disjoint set of variables. The set of terms over $\mathcal{F}$ and $\mathcal{V}$ is denoted by $\mathcal{T}(\mathcal{F}, \mathcal{V})$ and the set of ground terms over $\mathcal{F}$ by $\mathcal{T}(\mathcal{F})$. We write $\operatorname{Fun}(t)$ for the set of function symbols occurring in a term $t$. The size of a term $t$ is denoted $|t|$ and $\|t\|$ computes the number of occurrences of function symbols in $t$. A term $t$ is called linear if any variable $x$ occurs at most once in $t$. Positions are used to address symbol occurrences in terms. Given a term $t$ we use Pos $(t)$ to denote the set of positions induced by the term $t$ and we write $t(p)$ with $p \in \operatorname{Pos}(t)$ for the symbol at position $p$ in the term $t$. The subset of positions $p \in \operatorname{Pos}(t)$ such that $t(p) \in \mathcal{F}$ is denoted by $\operatorname{Pos}_{\mathcal{F}}(t)$.

A rewrite rule is a pair of terms $(l, r)$, written $l \rightarrow r$ such that $l$ is not a variable and all variables in $r$ are contained in $l$. A rewrite rule $l \rightarrow r$ is size-preserving (size-decreasing) if $|l|=|r|(|l|>|r|)$. A term rewrite system (TRS for short) is a set of rewrite rules. For complexity analysis we assume TRSs to be finite and terminating. A TRS $\mathcal{R}$ is said to be duplicating if there exist a rewrite rule $l \rightarrow r \in \mathcal{R}$ and a variable $x$ that occurs more often in $r$ than in $l$. A TRS $\mathcal{R}$ is called linear (left-linear, right-linear) if for all rewrite rules $l \rightarrow r \in \mathcal{R}$ the terms $l$ and $r(l, r)$ are linear. We call a TRS $\mathcal{R}$ collapsing if it contains a rewrite rule $l \rightarrow r$ such that $r$ is a variable. The defined symbols of a TRS $\mathcal{R}$ are all function symbols $f$ for which there is a rewrite rule $l \rightarrow r$ in $\mathcal{R}$ such that $f=l(\epsilon)$. In the following we denote this set of function symbols by $\operatorname{Def}(\mathcal{R})$. Those function symbols of $\mathcal{R}$ which are not defined are called constructor symbols. So the set of all constructor symbols is defined as $\operatorname{Con}(\mathcal{R})=\mathcal{F} \backslash \operatorname{Def}(\mathcal{R})$.

A rewrite relation is a binary relation on terms that is closed under contexts and substitutions. For a TRS $\mathcal{R}$ we define $\rightarrow_{\mathcal{R}}$ to be the smallest rewrite relation that contains $\mathcal{R}$. As usual $\rightarrow^{*}$ denotes the reflexive and transitive closure of $\rightarrow$ and $\rightarrow^{m}$ the $m$-th iterate of $\rightarrow$. A relative TRS $\mathcal{R} / \mathcal{S}$ is a pair of TRSs $\mathcal{R}$ and $\mathcal{S}$ with the induced rewrite relation $\rightarrow_{\mathcal{R} / \mathcal{S}}=\rightarrow_{\mathcal{S}}^{*} \cdot \rightarrow_{\mathcal{R}} \cdot \rightarrow_{\mathcal{S}}^{*}$. In the sequel we will sometimes identify a TRS $\mathcal{R}$ with the relative TRS $\mathcal{R} / \varnothing$ and vice versa. Furthermore properties defined for TRSs (as the ones above) naturally extend to relative TRSs.

The derivation height of a term $t$ with respect to a relation $\rightarrow$ is defined as follows: $\operatorname{dh}(t, \rightarrow)=\sup \left\{m \mid \exists u t \rightarrow^{m} u\right\}$. The complexity of a relation $\rightarrow$ with respect to a (possibly infinite) set of terms (or language) $L$, denoted by $\mathrm{cp}_{L}(n, \rightarrow)$, computes the maximal derivation height of all terms in $L$ up to size $n$ and is defined as $\mathrm{cp}_{L}(n, \rightarrow)=\sup \{\operatorname{dh}(t, \rightarrow) \mid$ $t \in L$ and $|t| \leqslant n\}$. Sometimes we say that a TRS $\mathcal{R}$ (relative TRS $\mathcal{R} / \mathcal{S}$ ) has linear, quadratic, etc. or polynomial complexity with respect to $L$ if $\mathrm{cp}_{L}\left(n, \rightarrow_{\mathcal{R}}\right)\left(\mathrm{cp}_{L}\left(n, \rightarrow_{\mathcal{R} / \mathcal{S}}\right)\right)$ can be bounded by a linear, quadratic, etc. function or polynomial in $n$. Let $\mathcal{R}$ be a TRS over some signature $\mathcal{F}$. The derivational complexity of $\mathcal{R}$, abbreviated by $\mathrm{dc}(n, \mathcal{R})$ and defined as $\operatorname{dc}(n, \mathcal{R})=\operatorname{cp}_{\mathcal{T}(\mathcal{F}, \mathcal{V})}\left(n, \rightarrow_{\mathcal{R}}\right)$, computes the complexity of $\rightarrow_{\mathcal{R}}$ with respect to all terms. In contrast, the runtime complexity of $\mathcal{R}$ considers the maximal derivation height of constructor-based terms only, i.e., $\mathrm{rc}(n, \mathcal{R})=\mathrm{cp}_{\mathcal{T}_{\text {con }}(\mathcal{R}, \mathcal{V})}\left(n, \rightarrow_{\mathcal{R}}\right)$. Here, the set of constructor-based terms $\mathcal{T}_{\text {Con }}(\mathcal{R}, \mathcal{V})$ is defined as the set of all terms $t=f\left(t_{1}, \ldots, t_{m}\right)$ such that $f \in \operatorname{Def}(\mathcal{R})$ and $t_{i} \in \mathcal{T}(\operatorname{Con}(\mathcal{R}), \mathcal{V})$ for all $i \in\{1, \ldots, m\}$.

For functions $f, g: \mathbb{N} \rightarrow \mathbb{N}$ we write $f(n)=\mathcal{O}(g(n))$ if there are constants $M, N \in \mathbb{N}$ such that $f(n) \leqslant M \cdot g(n)$ for all $n \geqslant N$. Furthermore, $f(n)=\Omega(g(n))$ if $g(n)=\mathcal{O}(f(n))$ and $f(n)=\Theta(g(n))$ if $f(n)=\mathcal{O}(g(n))$ and $f(n)=\Omega(g(n))$. 


\section{Modular Complexity via Relative Complexity}

In this section we present the basic idea that allows a modular treatment of complexity proofs. To this end we introduce complexity analysis for relative rewriting, i.e., given a relative TRS $\mathcal{R} / \mathcal{S}$ only the $\mathcal{R}$-steps contribute to the complexity. To estimate the derivational complexity of a relative TRS $\mathcal{R} / \mathcal{S}$, a pair of orderings $(\succ, \succeq)$ will be used such that $\mathcal{R} \subseteq \succ$ and $\mathcal{S} \subseteq \succeq$. The necessary properties of these orderings are given in the next definition.

Definition 3.1. A complexity pair $(\succ, \succeq)$ consists of two finitely branching rewrite relations $\succ$ and $\succeq$ that are compatible, i.e., $\succeq \cdot \succ \subseteq \succ$ and $\succ \cdot \succeq \subseteq \succ$. We call a relative TRS $\mathcal{R} / \mathcal{S}$ compatible with a complexity pair $(\succ, \succeq)$ if $\mathcal{R} \subseteq \succ$ and $\mathcal{S} \subseteq \succeq$.

The next lemma states that given a relative TRS $\mathcal{R} / \mathcal{S}$ and a compatible complexity pair $(\succ, \succeq)$, the $\succ$ ordering is crucial for estimating the derivational complexity of $\mathcal{R} / \mathcal{S}$. Intuitively the result states that every $\mathcal{R} / \mathcal{S}$-step gives rise to at least one $\succ$-step.

Lemma 3.2. Let $\mathcal{R} / \mathcal{S}$ be a relative $T R S$ compatible with a complexity pair $(\succ, \succeq)$. Then for any term $t$ we have $\mathrm{dh}(t, \succ) \geqslant \mathrm{dh}\left(t, \rightarrow_{\mathcal{R} / \mathcal{S}}\right)$.

Proof. By assumption $\mathcal{R} / \mathcal{S}$ is compatible with $(\succ, \succeq)$. Since $\succ$ and $\succeq$ are rewrite relations $\rightarrow_{\mathcal{R}} \subseteq \succ$ and $\rightarrow_{\mathcal{S}} \subseteq \succeq$ holds. From the compatibility of $\succ$ and $\succeq$ we obtain $\rightarrow_{\mathcal{R} / \mathcal{S}} \subseteq \succ$. Hence for any sequence

$$
t \rightarrow_{\mathcal{R} / \mathcal{S}} t_{1} \rightarrow_{\mathcal{R} / \mathcal{S}} t_{2} \rightarrow_{\mathcal{R} / \mathcal{S}} \cdots
$$

also

$$
t \succ t_{1} \succ t_{2} \succ \cdots
$$

holds. The result follows immediately from this.

Obviously $\succ$ must be at least well-founded if finite complexities should be estimated. Because we are especially interested in feasible upper bounds the following corollary is specialized to polynomials.

Corollary 3.3. Let $\mathcal{R} / \mathcal{S}$ be a relative TRS compatible with a complexity pair $(\succ, \succeq)$. If the complexity of $\succ$ with respect to some language $L$ is linear, quadratic, etc. or polynomial then the complexity of $\mathcal{R} / \mathcal{S}$ with respect to $L$ is linear, quadratic, etc. or polynomial.

Proof. By Lemma 3.2.

This corollary allows to investigate the complexity of (compatible) complexity pairs instead of the complexity of the underlying relative TRS. Sections 4 and 5 are dedicated to formulate powerful complexity pairs. A severe drawback of complexity pairs is that given a relative TRS $\mathcal{R} / \mathcal{S}$ all rules in $\mathcal{R}$ must be oriented strictly. In the following we present a modular approach which allows to combine different techniques for estimating the complexity of a relative TRS $\mathcal{R} / \mathcal{S}$ with respect to a language $L$. The fundamental idea is based on the following simple procedure. Instead of computing the complexity of $\mathcal{R} / \mathcal{S}$ at once we try to bound the complexity of $\mathcal{R} / \mathcal{S}$ by splitting $\mathcal{R}$ into smaller components $\mathcal{R}_{1}$ and $\mathcal{R}_{2}$. Here $\mathcal{R}=\mathcal{R}_{1} \cup \mathcal{R}_{2}$. The aim is to over-estimate $\operatorname{dh}\left(t, \rightarrow_{\mathcal{R} / \mathcal{S}}\right)$ by $\operatorname{dh}\left(t, \rightarrow_{\mathcal{R}_{1} /\left(\mathcal{R}_{2} \cup \mathcal{S}\right)}\right)+$ $\operatorname{dh}\left(t, \rightarrow_{\mathcal{R}_{2} /\left(\mathcal{R}_{1} \cup \mathcal{S}\right)}\right)$. For each relative TRS $\mathcal{R}_{i} /\left(\mathcal{R}_{3-i} \cup \mathcal{S}\right)$ with $i \in\{1,2\}$ we can proceed in two directions: we can either split up $\mathcal{R}_{i}$ into smaller components or over-estimate $\operatorname{dh}\left(t, \rightarrow_{\mathcal{R}_{i} /\left(\mathcal{R}_{3-i} \cup \mathcal{S}\right)}\right)$ by applying some suitable method. (Section 7 shows that this choice is performed automatically.) Finally the complexity of the original system is determined by 
summing up all intermediate results. The next lemma states the main observation in this direction.

Lemma 3.4. Let $\left(\mathcal{R}_{1} \cup \mathcal{R}_{2}\right) / \mathcal{S}$ be a relative $T R S$ and let $t$ be a terminating term. Then $\mathrm{dh}\left(t, \rightarrow_{\mathcal{R}_{1} /\left(\mathcal{R}_{2} \cup \mathcal{S}\right)}\right)+\mathrm{dh}\left(t, \rightarrow_{\mathcal{R}_{2} /\left(\mathcal{R}_{1} \cup \mathcal{S}\right)}\right) \geqslant \mathrm{dh}\left(t, \rightarrow\left(\mathcal{R}_{1} \cup \mathcal{R}_{2}\right) / \mathcal{S}\right)$.

Proof. We abbreviate $\mathcal{R}_{1} \cup \mathcal{R}_{2}$ by $\mathcal{R}$ and $\mathcal{R}_{3-i} \cup \mathcal{S}$ by $\mathcal{S}_{i}$ for $i \in\{1,2\}$. Assume that $\mathrm{dh}\left(t, \rightarrow_{\mathcal{R} / \mathcal{S}}\right)=m$. Then there exists a rewrite sequence

$$
t \rightarrow_{\mathcal{R} / \mathcal{S}} t_{1} \rightarrow_{\mathcal{R} / \mathcal{S}} t_{2} \rightarrow_{\mathcal{R} / \mathcal{S}} \cdots \rightarrow_{\mathcal{R} / \mathcal{S}} t_{m-1} \rightarrow_{\mathcal{R} / \mathcal{S}} t_{m}
$$

of length $m$. Next we investigate this sequence for every relative TRS $\mathcal{R}_{i} / \mathcal{S}_{i}(1 \leqslant i \leqslant 2)$ where $m_{i}$ overestimates how often rules from $\mathcal{R}_{i}$ have been applied in the original sequence. Fix $i$. If the sequence (11) does not contain an $\mathcal{R}_{i}$ step then $t \rightarrow_{\mathcal{S}_{i}}^{m} t_{m}$ and $m_{i}=0$. In the other case there exists a maximal (with respect to $m_{i}$ ) sequence

$$
t \rightarrow_{\mathcal{R}_{i} / \mathcal{S}_{i}} t_{j_{1}} \rightarrow_{\mathcal{R}_{i} / \mathcal{S}_{i}} t_{j_{2}} \rightarrow_{\mathcal{R}_{i} / \mathcal{S}_{i}} \cdots \rightarrow_{\mathcal{R}_{i} / \mathcal{S}_{i}} t_{j_{m_{i}-1}} \rightarrow_{\mathcal{R}_{i} / \mathcal{S}_{i}} t_{m}
$$

where $1 \leqslant j_{1}<j_{2}<\cdots<j_{m_{i}}=m$. Together with the fact that every rewrite rule in $\mathcal{R}$ is contained in $\mathcal{R}_{1}$ or $\mathcal{R}_{2}$ we have $m_{1}+m_{2} \geqslant m$. If $m_{i}=0$ we obviously have $\operatorname{dh}\left(t, \rightarrow_{\mathcal{R}_{i} / \mathcal{S}_{i}}\right) \geqslant m_{i}$ and if $t \rightarrow_{\mathcal{R}_{i} / \mathcal{S}_{i}}^{m_{i}} t_{m}$ with $m_{i}>0$ we know that $\operatorname{dh}\left(t, \rightarrow_{\mathcal{R}_{i} / \mathcal{S}_{i}}\right) \geqslant m_{i}$ by the choice of sequence (2). (Note that in both cases it can happen that $\operatorname{dh}\left(t, \rightarrow_{\mathcal{R}_{i} / \mathcal{S}_{i}}\right)>m_{i}$ because sequence (1) need not be maximal with respect to $\rightarrow_{\mathcal{R}_{i} / \mathcal{S}_{i}}$.) Putting things together yields

which concludes the proof.

$$
\operatorname{dh}\left(t, \rightarrow_{\mathcal{R}_{1} / \mathcal{S}_{1}}\right)+\operatorname{dh}\left(t, \rightarrow_{\mathcal{R}_{2} / \mathcal{S}_{2}}\right) \geqslant m_{1}+m_{2} \geqslant m=\operatorname{dh}\left(t, \rightarrow_{\mathcal{R} / \mathcal{S}}\right)
$$

As already indicated in the proof, the statement of the above lemma does not hold for equality. This is illustrated by the following example.

Example 3.5. Consider the relative TRS $\mathcal{R} / \mathcal{S}$ with $\mathcal{R}=\{\mathrm{a} \rightarrow \mathrm{b}, \mathrm{a} \rightarrow \mathrm{c}\}$ and $\mathcal{S}=\varnothing$. We have $\mathrm{a} \rightarrow_{\mathcal{R} / \mathcal{S}}$ b or $\mathrm{a} \rightarrow_{\mathcal{R} / \mathcal{S}}$ c. Hence $\operatorname{dh}\left(\mathrm{a}, \rightarrow_{\mathcal{R} / \mathcal{S}}\right)=1$. However, the sum of the derivation heights $\mathrm{dh}(\mathrm{a}, \rightarrow\{\mathrm{a} \rightarrow \mathrm{b}\} /\{\mathrm{a} \rightarrow \mathrm{c}\})$ and $\mathrm{dh}\left(\mathrm{a}, \rightarrow_{\{\mathrm{a} \rightarrow \mathrm{c}\} /\{\mathrm{a} \rightarrow \mathrm{b}\}}\right)$ is 2 .

Although for Lemma 3.4 equality cannot be established the next result states that for complexity analysis this does not matter.

Theorem 3.6. Let $\left(\mathcal{R}_{1} \cup \mathcal{R}_{2}\right) / \mathcal{S}$ be a relative $T R S$ and $L$ be a set of terminating terms. Then $\mathrm{cp}_{L}\left(n, \rightarrow\left(\mathcal{R}_{1} \cup \mathcal{R}_{2}\right) / \mathcal{S}\right)=\Theta\left(\mathrm{cp}_{L}\left(n, \rightarrow_{\mathcal{R}_{1} /\left(\mathcal{R}_{2} \cup \mathcal{S}\right)}\right)+\mathrm{cp}_{L}\left(n, \rightarrow_{\mathcal{R}_{2} /\left(\mathcal{R}_{1} \cup \mathcal{S}\right)}\right)\right)$.

Proof. We have to show that there are constants $M, N$ and $M^{\prime}, N^{\prime}$ such that for any term $t \in L$ the following two properties hold (for $N$ and $N^{\prime}$ choose 0 , i.e., a term $t$ being a normal form):

- $\operatorname{dh}\left(t, \rightarrow\left(\mathcal{R}_{1} \cup \mathcal{R}_{2}\right) / \mathcal{S}\right) \leqslant M \cdot\left(\operatorname{dh}\left(t, \rightarrow_{\mathcal{R}_{1} /\left(\mathcal{R}_{2} \cup \mathcal{S}\right)}\right)+\operatorname{dh}\left(t, \rightarrow_{\mathcal{R}_{2} /\left(\mathcal{R}_{1} \cup \mathcal{S}\right)}\right)\right)$

- $M^{\prime} \cdot \operatorname{dh}\left(t, \rightarrow\left(\mathcal{R}_{1} \cup \mathcal{R}_{2}\right) / \mathcal{S}\right) \geqslant \operatorname{dh}\left(t, \rightarrow_{\mathcal{R}_{1} /\left(\mathcal{R}_{2} \cup \mathcal{S}\right)}\right)+\operatorname{dh}\left(t, \rightarrow_{\mathcal{R}_{2} /\left(\mathcal{R}_{1} \cup \mathcal{S}\right)}\right)$

The result then follows from this. Lemma 3.4 shows the first property with $M=1$. For the second property we reason as follows. Let $i \in\{1,2\}$ and $\mathcal{S}_{i}=\mathcal{R}_{3-i} \cup \mathcal{S}$. Since $t \rightarrow_{\mathcal{R}_{i} / \mathcal{S}_{i}} t^{\prime}$

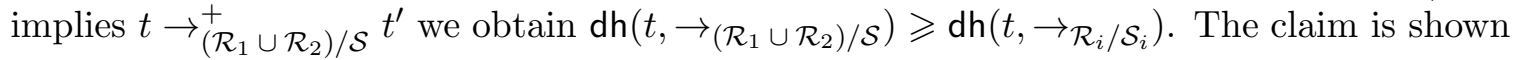
by choosing $M^{\prime}=2$. 


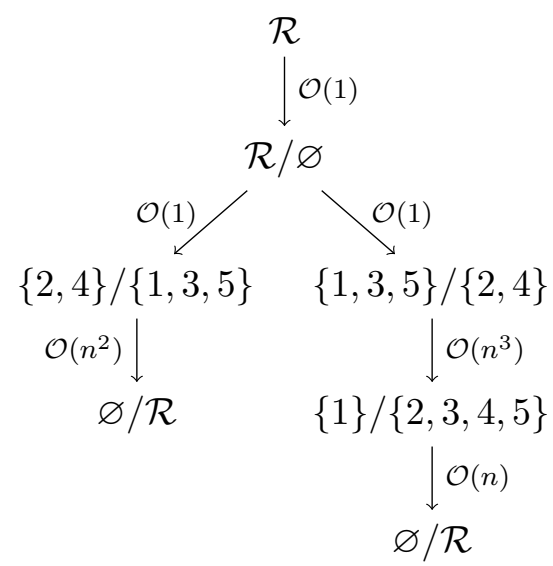

FiguRE 1. Sketch of a modular complexity proof

Theorem 3.6 allows to split a relative TRS $\left(\mathcal{R}_{1} \cup \mathcal{R}_{2}\right) / \mathcal{S}$ into smaller components $\mathcal{R}_{1} /\left(\mathcal{R}_{2} \cup \mathcal{S}\right)$ and $\mathcal{R}_{2} /\left(\mathcal{R}_{1} \cup \mathcal{S}\right)$ and evaluate the complexities of these components (e.g., by different complexity pairs) independently. Note that this approach is not restricted to relative rewriting. To estimate the complexity of a (non-relative) TRS $\mathcal{R}$ just consider the relative TRS $\mathcal{R} / \varnothing$. The next example shows how proofs in the modular framework look like. Section 7 gives more details on proof trees.

Example 3.7. Proofs in the modular setting can be viewed as trees. We sketch such a proof in Figure 1 using the TRS $\mathcal{R}$ consisting of the following five rules:

$$
\begin{array}{rlrl}
1: & & \operatorname{rev}(x) & \rightarrow \operatorname{rev}^{\prime}(x, \operatorname{nil}) \\
2: & \operatorname{rev}^{\prime}(\operatorname{nil}, y) & \rightarrow y \\
3: & \operatorname{rev}^{\prime}(\operatorname{cons}(x, y), z) & \rightarrow \operatorname{rev}^{\prime}(y, \operatorname{append}(\operatorname{cons}(x, \operatorname{nil}), z)) \\
\text { 4: } & & \operatorname{append}(\operatorname{nil}, y) & \rightarrow y \\
\text { 5: } & \text { append }(\operatorname{cons}(x, y), z) & \rightarrow \operatorname{cons}(x, \operatorname{append}(y, z))
\end{array}
$$

The root node of the tree is the TRS of interest and the other nodes are relative rewrite systems representing intermediate complexity problems. The edges indicate the (derivational) complexity of the proof steps. It is possible to apply Theorem 3.6 explicitly to split a problem into two (or more) problems as demonstrated in the second node. Such situations do not affect the complexity of the given problem which justifies the labels $\mathcal{O}(1)$. The remaining proof steps measure the complexity of the rewrite rules that are moved from the first into the second component (relative to the remaining rules). These steps rely on an implicit application of Theorem 3.6. For instance in the proof tree shown in Figure 1 there is an edge from $\{1,3,5\} /\{2,4\}$ to $\{1\} /\{2,3,4,5\}$ labeled $\mathcal{O}\left(n^{3}\right)$, stating that the (derivational) complexity of $\{3,5\} /\{1,2,4\}$ is at most cubic. This step is sound because from Theorem 3.6 we know that computing an upper bound on $\{1\} /\{2,3,4,5\}$ and $\{3,5\} /\{1,2,4\}$ suffice to get a valid upper bound on $\{1,3,5\} /\{2,4\}$. In Sections 4 and 5 we study criteria that allow to perform such proof steps. Since the leaves in the tree give rise to constant complexity, the complexity of the original problem can be overestimated by summing up the complexities annotated to the edges; yielding a cubic upper bound in this exemplary case. Later (Example 7.11) we will see that this bound is not tight. 
In the next two sections we study how matrix interpretations and the match-bounds technique can be suited for relative complexity analysis.

\section{Matrix Interpretations}

This section is aimed at formulating complexity pairs based on matrix interpretations [6]. Since our interest is in polynomial upper bounds, triangular matrix interpretations [20] and arctic matrix interpretations [15] are considered. The last part of this section generalizes the weight gap principle from [11] to (a restriction of) triangular matrix interpretations and relative rewriting.

4.1. Preliminaries. An $\mathcal{F}$-algebra $\mathcal{A}$ consists of a non-empty carrier $A$ and a set of interpretations $f_{\mathcal{A}}$ for every $f \in \mathcal{F}$. By $[\alpha]_{\mathcal{A}}(\cdot)$ we denote the usual evaluation function of $\mathcal{A}$ according to an assignment $\alpha$. An $\mathcal{F}$-algebra $\mathcal{A}$ together with two relations $\succ$ and $\succeq$ on $A$ is called a monotone algebra if every $f_{\mathcal{A}}$ is monotone with respect to $\succ$ and $\succeq, \succ$ is a well-founded order, and $\succ$ and $\succeq$ are compatible. Any monotone algebra $(\mathcal{A}, \succ, \succeq)$ induces a well-founded order on terms, i.e., $s \succ_{\mathcal{A}} t$ if for any assignment $\alpha$ the condition $[\alpha]_{\mathcal{A}}(s) \succ[\alpha]_{\mathcal{A}}(t)$ holds. The order $\succeq_{\mathcal{A}}$ is defined similarly. A relative TRS $\mathcal{R} / \mathcal{S}$ is compatible with a monotone algebra $(\mathcal{A}, \succ, \succeq)$ if $\mathcal{R} / \mathcal{S}$ is compatible with $\left(\succ_{\mathcal{A}}, \succeq_{\mathcal{A}}\right)$.

4.2. Triangular Matrix Interpretations. Matrix interpretations $(\mathcal{M}, \succ, \succeq$ ) (often just denoted $\mathcal{M}$ ) are a special form of monotone algebras. Here the carrier is $\mathbb{N}^{d}$ for some fixed dimension $d \in \mathbb{N} \backslash\{0\}$. The order $\succeq$ is the point-wise extension of $\geqslant_{\mathbb{N}}$ to vectors and $\vec{u} \succ \vec{v}$ if $u_{1}>_{\mathbb{N}} v_{1}$ and $\vec{u} \succeq \vec{v}$. If every $f \in \mathcal{F}$ of arity $n$ is interpreted as $f_{\mathcal{M}}\left(\overrightarrow{x_{1}}, \ldots, \overrightarrow{x_{n}}\right)=$ $F_{1} \overrightarrow{x_{1}}+\cdots+F_{n} \overrightarrow{x_{n}}+\vec{f}$ where $F_{i} \in \mathbb{N}^{d \times d}$ for all $1 \leqslant i \leqslant n$ and $\vec{f} \in \mathbb{N}^{d}$ then monotonicity of $\succ$ is achieved by demanding $F_{i(1,1)} \geqslant 1$ for any $f \in \mathcal{F}$ and $1 \leqslant i \leqslant n$. Such interpretations have been introduced in [6].

A matrix interpretation where for every $f \in \mathcal{F}$ all $F_{i}(1 \leqslant i \leqslant n$ where $n$ is the arity of $f$ ) are upper triangular is called triangular matrix interpretation (abbreviated by TMI). A square matrix $A$ of dimension $d$ is of upper triangular shape if $A_{(i, i)} \leqslant 1$ and $A_{(i, j)}=0$ if $i>j$ for all $1 \leqslant i, j \leqslant d$. For historic reasons a TMI based on matrices of dimension one is also called strongly linear interpretation (SLI for short). In [20] it is shown that the derivational complexity of a TRS $\mathcal{R}$ is bounded by a polynomial of degree $d$ if there exists a TMI $\mathcal{M}$ of dimension $d$ such that $\mathcal{R} \subseteq \succ_{\mathcal{M}}$. For our setting the following formulation is more useful.

Theorem 4.1. Let $\mathcal{M}$ be a TMI of dimension d over a signature $\mathcal{F}$. Then $\left(\succ_{\mathcal{M}}, \succeq_{\mathcal{M}}\right)$ is a complexity pair. Furthermore $\mathrm{cp}_{\mathcal{T}(\mathcal{F}, \mathcal{V})}\left(n, \succ_{\mathcal{M}}\right)=\mathcal{O}\left(n^{d}\right)$.

Proof. Straightforward from [20, Theorem 6]. 
The following example familiarizes the reader with TMIs.

Example 4.2. Consider the relative TRS $\mathcal{R} / \mathcal{S}$ over the signature $\mathcal{F}=\{\mathrm{f}, \mathrm{g}\}$ defined as $\mathcal{R}=\{\mathrm{f}(\mathrm{f}(x)) \rightarrow \mathrm{f}(\mathrm{g}(\mathrm{f}(x)))\}$ and $\mathcal{S}=\{\mathrm{f}(x) \rightarrow x\}$. Then the TMI $\mathcal{M}$ of dimension two with

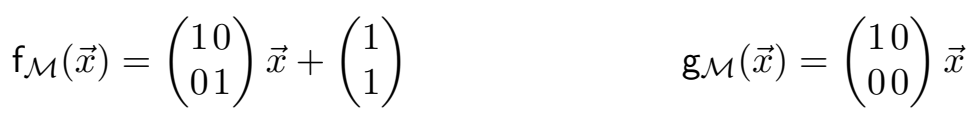

induces the complexity pair $\left(\succ_{\mathcal{M}}, \succeq_{\mathcal{M}}\right)$. Furthermore $\mathcal{R} / \mathcal{S}$ is compatible with $\left(\succ_{\mathcal{M}}, \succeq_{\mathcal{M}}\right)$. Theorem 4.1 gives a quadratic upper bound on $\operatorname{cp}_{\mathcal{T}(\mathcal{F}, \mathcal{V})}\left(n, \succ_{\mathcal{M}}\right)$. Hence the derivational complexity of $\mathcal{R} / \mathcal{S}$ is at most quadratic by Corollary 3.3. It is easy to see (cf. Example 4.4) that this bound is not tight. We remark that there cannot exist an SLI that establishes a linear upper bound because no SLI can orient the rule $\mathrm{f}(\mathrm{f}(x)) \rightarrow \mathrm{f}(\mathrm{g}(\mathrm{f}(x)))$ strictly.

4.3. Arctic Matrix Interpretations. We define $\mathbb{A}=\mathbb{N} \cup\{-\infty\}$. For matrices $A \in$ $\mathbb{A}^{n \times m}$ and $B \in \mathbb{A}^{m \times p}$ the operation $\otimes$ yields an $n \times p$ matrix and is defined as follows: $(A \otimes B)_{(i, j)}=\max _{1 \leqslant k \leqslant m}\left\{A_{(i, k)}+B_{(k, j)}\right\}$ where + and max are extended naturally to deal with $-\infty$ (see [15]). Furthermore $x>_{\mathbb{A}} y$ if and only if $x>_{\mathbb{N}} y$ or $x=y=-\infty$, and $x \geqslant_{\mathbb{A}} y$ if and only if $x \geqslant_{\mathbb{N}} y$ or $y=-\infty .2$

An arctic matrix interpretation $(\mathcal{A}, \succ, \succeq$ ) (abbreviated by AMI and often just denoted $\mathcal{A}$ ) is a special form of a monotone algebra. Here the carrier is $\mathbb{A}^{d}$ for some fixed dimension $d \in \mathbb{N} \backslash\{0\}$. The orders $\succeq$ and $\succ$ are the point-wise extensions of $\geqslant_{\mathbb{A}}$ and $>_{\mathbb{A}}$ to vectors, respectively. Every unary function symbol $f \in \mathcal{F}$ is interpreted as $f_{\mathcal{A}}(\vec{x})=F \otimes \vec{x}$ where $F \in \mathbb{A}^{d \times d}$ and every constant $c$ as $c_{\mathcal{A}}=\vec{c}$ where $\vec{c} \in \mathbb{A}^{d}$. Monotonicity of $\succ$ is achieved by the restriction to at most unary function symbols and by demanding that $F_{(1,1)}$ and $c_{1}$ are different from $-\infty$ for unary function symbols $f$ and constants $c$, respectively. In [15] it is shown that the derivational complexity of a TRS $\mathcal{R}$, which contains unary and constant function symbols only, is at most linear if there exists an AMI $\mathcal{A}$ (of some dimension $d$ ) such that $\mathcal{R} \subseteq \succ_{\mathcal{A}}$.

Theorem 4.3. Let $\mathcal{A}$ be an $A M I$ of dimension $d$ over a signature $\mathcal{F}$ that contains constants and unary function symbols only. Then $\left(\succ_{\mathcal{A}}, \succeq_{\mathcal{A}}\right)$ is a complexity pair. Furthermore $\mathrm{cp}_{\mathcal{T}(\mathcal{F}, \mathcal{V})}\left(n, \succ_{\mathcal{A}}\right)=\mathcal{O}(n)$.

Proof. Straightforward from [15, Lemma 17].

Example 4.4. Consider the TRSs from Example 4.2, Then the AMI $\mathcal{A}$ satisfying

$$
\mathrm{f}_{\mathcal{A}}(\vec{x})=\left(\begin{array}{c}
13 \\
03
\end{array}\right) \vec{x} \quad \mathrm{~g}_{\mathcal{A}}(\vec{x})=\left(\begin{array}{cc}
0 & 1 \\
-\infty & -\infty
\end{array}\right) \vec{x}
$$

induces the complexity pair $\left(\succ_{\mathcal{A}}, \succeq_{\mathcal{A}}\right)$. Furthermore $\mathcal{R} / \mathcal{S}$ is compatible with $\left(\succ_{\mathcal{A}}, \succeq_{\mathcal{A}}\right)$. Theorem 4.3 gives a linear upper bound on $\operatorname{cp}_{\mathcal{T}(\mathcal{F}, \mathcal{V})}\left(n, \succ_{\mathcal{A}}\right)$. Hence the derivational complexity of $\mathcal{R} / \mathcal{S}$ is at most linear by Corollary [3.3. It is easy to see that this bound is tight.

\footnotetext{
${ }^{2}$ Note that $-\infty>_{\mathbb{A}}-\infty$ and hence $>_{\mathbb{A}}$ is not well-founded. Hence such comparisons are disallowed at certain matrix positions.
} 
4.4. Complexity Gap Principle. An obvious question is whether it suffices to estimate polynomial complexity of $\left(\mathcal{R}_{1} \cup \mathcal{R}_{2}\right) / \mathcal{S}$ by establishing polynomial upper bounds on the complexities of $\mathcal{R}_{1} /\left(\mathcal{R}_{2} \cup \mathcal{S}\right.$ ) and $\mathcal{R}_{2} / \mathcal{S}$ (in contrast to $\mathcal{R}_{2} /\left(\mathcal{R}_{1} \cup \mathcal{S}\right.$ ) as in Theorem 3.6). The following example by Hofbauer [14] shows that in general the complexity of $\left(\mathcal{R}_{1} \cup \mathcal{R}_{2}\right) / \mathcal{S}$ might be much larger than the sum of the components above; even for systems where both parts have linear complexity. Here $\mathcal{S}=\varnothing$.

Example 4.5. Consider the TRS $\mathcal{R}_{1}$ consisting of the single rule

$$
\mathrm{c}(\mathrm{L}(x)) \rightarrow \mathrm{R}(x)
$$

and the TRS $\mathcal{R}_{2}$ consisting of the rewrite rules

$$
\mathrm{R}(\mathrm{a}(x)) \rightarrow \mathrm{b}(\mathrm{b}(\mathrm{R}(x))) \quad \mathrm{R}(x) \rightarrow \mathrm{L}(x) \quad \mathrm{b}(\mathrm{L}(x)) \rightarrow \mathrm{L}(\mathrm{a}(x))
$$

The derivational complexity of the relative TRS $\mathcal{R}_{1} / \mathcal{R}_{2}$ is linear, due to the SLI that just counts the c's. The derivational complexity of $\mathcal{R}_{2}$ is linear as well since the system can be proved terminating by the match-bound technique [8]. However, the TRS $\mathcal{R}_{1} \cup \mathcal{R}_{2}$ admits exponentially long derivations in the size of the starting term:

$$
\begin{aligned}
\mathrm{c}^{n}(\mathrm{~L}(\mathrm{a}(x))) & \rightarrow \mathrm{c}^{n-1}(\mathrm{R}(\mathrm{a}(x))) \rightarrow \mathrm{c}^{n-1}(\mathrm{~b}(\mathrm{~b}(\mathrm{R}(x)))) \rightarrow \mathrm{c}^{n-1}(\mathrm{~b}(\mathrm{~b}(\mathrm{~L}(x)))) \\
& \rightarrow \mathrm{c}^{n-1}(\mathrm{~b}(\mathrm{~L}(\mathrm{a}(x)))) \rightarrow \mathrm{c}^{n-1}(\mathrm{~L}(\mathrm{a}(\mathrm{a}(x)))) \rightarrow{ }^{*} \mathrm{~L}\left(\mathrm{a}^{2^{n}}(x)\right)
\end{aligned}
$$

Under certain circumstances the problem of the preceding example does not occur. Inspired by the weight gap principle of Hirokawa and Moser [11] (which was developed to estimate weak dependency pair steps relative to usable rule steps), below we state abstract criteria on $\mathcal{R}_{1}$ and $\mathcal{R}_{2}$ such that the complexity of $\mathcal{R}_{1} /\left(\mathcal{R}_{2} \cup \mathcal{S}\right)$ and $\mathcal{R}_{2} / \mathcal{S}$ determines the complexity of $\left(\mathcal{R}_{1} \cup \mathcal{R}_{2}\right) / \mathcal{S}$.

Theorem 4.6 (Complexity Gap Principle). Let $\left(\mathcal{R}_{1} \cup \mathcal{R}_{2}\right) / \mathcal{S}$ be a relative $T R S$ and $L$ be a set of terminating terms. If there exist a complexity pair $(\succ, \succeq)$ and a constant $\Delta$ such that $\mathcal{R}_{2} / \mathcal{S}$ is compatible with $(\succ, \succeq)$ and $u \rightarrow_{\mathcal{R}_{1}} v$ implies $\operatorname{dh}(u, \succ)+\Delta \geqslant \operatorname{dh}(v, \succ)$ then $\mathrm{cp}_{L}\left(n, \rightarrow\left(\mathcal{R}_{1} \cup \mathcal{R}_{2}\right) / \mathcal{S}\right)=\mathcal{O}\left(\mathrm{cp}_{L}\left(n, \rightarrow_{\mathcal{R}_{1} /\left(\mathcal{R}_{2} \cup \mathcal{S}\right)}\right)+\mathrm{cp}_{L}(n, \succ)\right)$.

Proof. We show that under the above assumptions, for any term $s \in L$ there exists a constant $M$ such that $\operatorname{dh}\left(s, \rightarrow\left(\mathcal{R}_{1} \cup \mathcal{R}_{2}\right) / \mathcal{S}\right) \leqslant M \cdot \operatorname{dh}\left(s, \rightarrow_{\mathcal{R}_{1} /\left(\mathcal{R}_{2} \cup \mathcal{S}\right)}\right)+\operatorname{dh}(s, \succ)$. Consider a derivation of maximal length in $\left(\mathcal{R}_{1} \cup \mathcal{R}_{2}\right) / \mathcal{S}$, written as follows:

$$
s=s_{0} \rightarrow_{\mathcal{R}_{2} / \mathcal{S}}^{k_{0}} \cdot \rightarrow_{\mathcal{S}}^{*} t_{0} \rightarrow_{\mathcal{R}_{1}} s_{1} \rightarrow_{\mathcal{R}_{2} / \mathcal{S}}^{k_{1}} \cdot \rightarrow_{\mathcal{S}}^{*} t_{1} \rightarrow_{\mathcal{R}_{1}} \cdots \rightarrow_{\mathcal{R}_{1}} s_{m} \rightarrow_{\mathcal{R}_{2} / \mathcal{S}}^{k_{m}} \cdot \rightarrow_{\mathcal{S}}^{*} t_{m}
$$

Since sequence (11) is maximal, $\operatorname{dh}\left(s_{0}, \rightarrow\left(\mathcal{R}_{1} \cup \mathcal{R}_{2}\right) / \mathcal{S}\right) \leqslant \mathrm{dh}\left(s_{0}, \rightarrow_{\mathcal{R}_{1} /\left(\mathcal{R}_{2} \cup \mathcal{S}\right)}\right)+\sum_{0 \leqslant i \leqslant m} k_{i}$. Because $\mathcal{R}_{2} / \mathcal{S}$ is compatible with $(\succ, \succeq)$ we have $\operatorname{dh}\left(s_{0}, \succ\right) \geqslant \operatorname{dh}\left(t_{0}, \succ\right)+k_{0}$. From the assumption, $\operatorname{dh}\left(t_{0}, \succ\right)+\Delta \geqslant \operatorname{dh}\left(s_{1}, \succ\right)$ follows and hence $\operatorname{dh}\left(s_{0}, \succ\right)+\Delta \geqslant \operatorname{dh}\left(s_{1}, \succ\right)+k_{0}$. Repeating this argument shows $\operatorname{dh}\left(s_{0}, \succ\right)+m \cdot \Delta \geqslant \sum_{0 \leqslant i \leqslant m} k_{i}$. Because $m \leqslant \operatorname{dh}\left(s_{0}, \rightarrow_{\mathcal{R}_{1} /\left(\mathcal{R}_{2} \cup \mathcal{S}\right)}\right)$ (note that equality does not hold since sequence (11) need not be maximal for $\mathcal{R}_{1} /\left(\mathcal{R}_{2} \cup \mathcal{S}\right)$ ) we obtain $\operatorname{dh}\left(s_{0}, \rightarrow\left(\mathcal{R}_{1} \cup \mathcal{R}_{2}\right) / \mathcal{S}\right) \leqslant \operatorname{dh}\left(s_{0}, \rightarrow_{\mathcal{R}_{1} /\left(\mathcal{R}_{2} \cup \mathcal{S}\right)}\right)+\operatorname{dh}\left(s_{0}, \succ\right)+\operatorname{dh}\left(s_{0}, \rightarrow_{\mathcal{R}_{1} /\left(\mathcal{R}_{2} \cup \mathcal{S}\right)}\right) \cdot \Delta$ which simplifies to $\operatorname{dh}\left(s_{0}, \rightarrow\left(\mathcal{R}_{1} \cup \mathcal{R}_{2}\right) / \mathcal{S}\right) \leqslant(\Delta+1) \cdot \operatorname{dh}\left(s_{0}, \rightarrow_{\mathcal{R}_{1} /\left(\mathcal{R}_{2} \cup \mathcal{S}\right)}\right)+\operatorname{dh}\left(s_{0}, \succ\right)$. Finally, taking $M=\Delta+1$ concludes the proof. 
To implement the above theorem the question arises which further requirements besides compatibility of $\mathcal{R}_{2} / \mathcal{S}$ with a complexity pair $(\succ, \succeq)$ are required such that for any terms $u$ and $v$ a step $u \rightarrow_{\mathcal{R}_{1}} v$ implies the desired $\operatorname{dh}(u, \succ)+\Delta \geqslant \operatorname{dh}(s, \succ)$ for some constant $\Delta$. One idea is to test $\operatorname{dh}(l, \succ)+\Delta \geqslant \operatorname{dh}(r, \succ)$ explicitly for any $l \rightarrow r \in \mathcal{R}_{1}$ and demand that the complexity pair $(\succ, \succeq)$ then satisfies $\operatorname{dh}(C[l \sigma], \succ)+\Delta \geqslant \operatorname{dh}(C[r \sigma], \succ)$ for all contexts $C$ and substitutions $\sigma$.

As we know from [11, SLIs can be used to get a concrete instance of Theorem 4.6 with respect to derivational complexity, if $\mathcal{S}$ is empty. Below we state the result in the relative setting, which is more useful for our purposes.

Corollary 4.7. Let $\left(\mathcal{R}_{1} \cup \mathcal{R}_{2}\right) / \mathcal{S}$ be a relative $T R S, \mathcal{R}_{1}$ be non-duplicating, and $\mathcal{R}_{2} / \mathcal{S}$ be compatible with an $S L I$. Then $\mathrm{dc}\left(n,\left(\mathcal{R}_{1} \cup \mathcal{R}_{2}\right) / \mathcal{S}\right)=\mathcal{O}\left(\mathrm{dc}\left(n, \mathcal{R}_{1} /\left(\mathcal{R}_{2} \cup \mathcal{S}\right)\right)+n\right)$.

Proof. Follows from Theorems 4.6 and 4.1 using the complexity pair $\left(\succ_{\mathcal{M}}, \succeq_{\mathcal{M}}\right)$ induced by the SLI $\mathcal{M}$.

An immediate consequence of the above corollary is that for any relative TRS $\mathcal{R} / \mathcal{S}$ we can shift rewrite rules in $\mathcal{R}$ that are strictly oriented by an SLI $\mathcal{M}$ into the $\mathcal{S}$-component, provided that $\mathcal{R}$ is non-duplicating and all rules in $\mathcal{S}$ behave nicely with respect to $\succeq_{\mathcal{M}}$. Note that the above corollary does not require that all rules from $\mathcal{R}$ are (strictly) oriented. This causes some kind of non-determinism which is demonstrated in the next example.

Example 4.8. Consider the TRS (Bouchare_06/12) 3 consisting of the rules:

$$
1: \mathrm{b}(\mathrm{b}(x) \rightarrow \mathrm{a}(\mathrm{a}(\mathrm{a}(x))) \quad 2: \mathrm{b}(\mathrm{a}(\mathrm{b}(x))) \rightarrow \mathrm{a}(x) \quad 3: \mathrm{b}(\mathrm{a}(\mathrm{a}(x))) \rightarrow \mathrm{b}(\mathrm{a}(\mathrm{b}(x)))
$$

The SLI $\mathcal{M}$ with $\mathrm{a}_{\mathcal{M}}(x)=x+2$ and $\mathrm{b}_{\mathcal{M}}(x)=x+1$ transforms the TRS into $\{1\} /\{2,3\}$ which is compatible with the AMI $\mathcal{A}$ (where all matrix coefficients are smaller than two)

$$
\mathrm{a}_{\mathcal{A}}(\vec{x})=\left(\begin{array}{ccc}
0 & 0 & 0 \\
-\infty & -\infty & 0 \\
-\infty & 0 & -\infty
\end{array}\right) \vec{x} \quad \mathrm{~b}_{\mathcal{A}}(\vec{x})=\left(\begin{array}{ccc}
0 & 1 & 0 \\
0 & 1 & 0 \\
-\infty & 0 & -\infty
\end{array}\right) \vec{x}
$$

showing linear derivational complexity of this TRS. If a different SLI is used in the first step, e.g., the one that counts just b's then the intermediate problem $\{3\} /\{1,2\}$ remains to be solved. For this problem there exists no AMI of dimension three where all entries are less than 2 (but there exists one where all entries are less than 3). For an implementation this means that depending on the rules the SLI orients, later techniques may succeed or fail.

Next we remark on another subtlety of Theorem 4.6. Assume that $\mathcal{R}_{1} /\left(\mathcal{R}_{2} \cup \mathcal{S}\right)$ is compatible with a complexity pair $(\succ, \succeq)$. Then $\left(\mathcal{R}_{1} \cup \mathcal{R}_{2}\right) / \mathcal{S}$ is transformed into the problem $\mathcal{R}_{2} /\left(\mathcal{R}_{1} \cup \mathcal{S}\right)$ and this proof step estimates the complexity of $\mathcal{R}_{1} /\left(\mathcal{R}_{2} \cup \mathcal{S}\right)$. If the complexity gap principle is used the situation changes. Since it does not require (weak) compatibility with $\mathcal{R}_{1}$, it does not make a statement about the complexity of $\mathcal{R}_{1} /\left(\mathcal{R}_{2} \cup \mathcal{S}\right)$. Instead it states that the complexity of $\left(\mathcal{R}_{1} \cup \mathcal{R}_{2}\right) / \mathcal{S}$ is dominated by the complexity of $\mathcal{R}_{1} /\left(\mathcal{R}_{2} \cup \mathcal{S}\right)$ or the complexity of $\mathcal{R}_{2} / \mathcal{S}$. This behavior is illustrated in the next example.

Example 4.9. Consider the relative TRS $\mathcal{R}$ consisting of the two rules

$$
1: \mathrm{c}(x) \rightarrow \mathrm{a}(x) \quad 2: \mathrm{a}(\mathrm{b}(x)) \rightarrow \mathrm{b}(\mathrm{b}(\mathrm{c}(x)))
$$

\footnotetext{
${ }^{3}$ Labels in sans-serif font refer to TRSs from the TPDB 7.0.2, see http://termination-portal.org
} 
We observe that the derivational complexity of the TRS $\mathcal{R}$ is at least exponential because

$$
\mathrm{a}^{n}(\mathrm{~b}(x)) \rightarrow^{2} \mathrm{a}^{n-1}(\mathrm{~b}(\mathrm{~b}(\mathrm{a}(x)))) \rightarrow^{4} \mathrm{a}^{n-2}(\mathrm{~b}(\mathrm{~b}(\mathrm{~b}(\mathrm{~b}(\mathrm{a}(x)))))) \rightarrow^{8} \cdots \rightarrow^{2^{n}} \mathrm{~b}^{2^{n}}(\mathrm{a}(x))
$$

Obviously both rules are applied exponentially often in this sequence. Nevertheless by an SLI that counts c's Corollary 4.7 can be applied to $\mathcal{R}$ to obtain the relative TRS $\{2\} /\{1\}$. As remarked earlier this step does not yield an upper bound on the complexity of the TRS $\{1\} /\{2\}$ but only on the TRS $\{1\}$.

Next we give counterexamples that TMIs, AMIs, and match-bounds cannot be used to implement Theorem 4.6. A suitable but severe restriction of TMIs is considered in Theorem 4.11

Examples 4.10. Recall the two TRSs $\mathcal{R}_{1}$ and $\mathcal{R}_{2}$ from Example 4.5, Here $\mathcal{S}=\varnothing$. Since $\mathrm{dc}\left(n, \mathcal{R}_{1} / \mathcal{R}_{2}\right)=\mathcal{O}(n)$ and $\mathrm{dc}\left(n, \mathcal{R}_{1} \cup \mathcal{R}_{2}\right)=\Omega\left(2^{n}\right)$ any method that establishes $\mathrm{dc}\left(n, \mathcal{R}_{2}\right)=$ $\mathcal{O}\left(n^{k}\right)$ for some $k \in \mathbb{N}$ cannot be used to implement the complexity gap principle.

Since the TMI $\mathcal{M}$ with

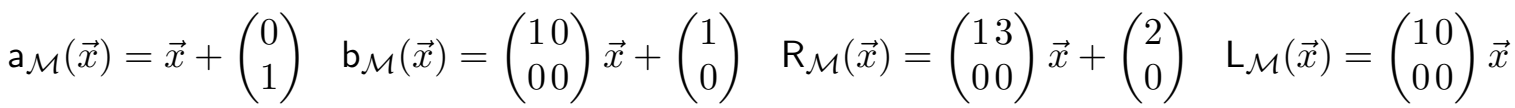

orients all rules in $\mathcal{R}_{2}$ strictly — and hence gives a quadratic upper bound on $\mathrm{dc}\left(n, \mathcal{R}_{2}\right)$-in general TMIs cannot adhere to Theorem 4.6. The problem for the interpretation above is that although there exists a $\Delta$ with $\operatorname{dh}(l, \succ)+\Delta \geqslant \operatorname{dh}(r, \succ)$ for all $l \rightarrow r \in \mathcal{R}_{1}$ this property is not closed under substitutions. (The situation is different, however, if the matrix interpretation has constant growth, see Theorem 4.11 below.)

Similarly, the AMI $\mathcal{A}$ (inducing at most linear derivational complexity of $\mathcal{R}_{2}$ ) with

$$
\mathrm{a}_{\mathcal{A}}(\vec{x})=\left(\begin{array}{cc}
0 & -\infty \\
3 & 3
\end{array}\right) \vec{x} \quad \mathrm{~b}_{\mathcal{A}}(\vec{x})=\left(\begin{array}{cc}
1 & 2 \\
-\infty & 0
\end{array}\right) \vec{x} \quad \mathrm{R}_{\mathcal{A}}(\vec{x})=\left(\begin{array}{c}
13 \\
02
\end{array}\right) \vec{x} \quad \mathrm{~L}_{\mathcal{A}}(\vec{x})=\left(\begin{array}{cc}
0 & -\infty \\
-\infty & -\infty
\end{array}\right) \vec{x}
$$

violates the same requirement in Theorem 4.6 as the TMI $\mathcal{M}$ above.

A similar reasoning also holds for match-bounds; one easily verifies that match-bounds apply to the TRS $\mathcal{R}_{2}$ and hence this system admits linear derivational complexity. The problem in this setting is that a valid termination proof of $\mathcal{R}_{2}$ using match-bounds does not necessarily yield a rewrite relation $\succ$ such that $\operatorname{dh}(u, \succ)+\Delta \geqslant \operatorname{dh}(v, \succ)$ whenever $u \rightarrow_{\mathcal{R}_{1}} v$, as required by Theorem 4.6 .

Finally we present a criterion that allows to implement Theorem 4.6 based on TMIs. To this end we introduce the following concepts. A matrix interpretation $\mathcal{M}$ has constant growth if there is a matrix $A$ such that for any $p \in \mathbb{N}$ and matrices $M_{1}, \ldots, M_{p}$ in $\mathcal{M}$ we have $M_{1} \cdot \ldots \cdot M_{p} \leqslant A$. Here $\leqslant$ is the pointwise extension of $\leqslant \mathbb{N}$ to matrices. Because of the shape of matrix interpretations for terms $s$ and $t$ there exist $k \in \mathbb{N}$, matrices $S_{1}, \ldots, S_{k}, T_{1}, \ldots, T_{k}$, and vectors $\vec{s}, \vec{t}$ such that $[\alpha]_{\mathcal{M}}(s)=S_{1} \alpha\left(x_{1}\right)+\cdots+S_{k} \alpha\left(x_{k}\right)+\vec{s}$ and $[\alpha]_{\mathcal{M}}(t)=T_{1} \alpha\left(x_{1}\right)+$ $\cdots+T_{k} \alpha\left(x_{k}\right)+\vec{t}$. In such a case we denote the non-constant part of the interpretation of $s$ by $[\alpha]_{\mathcal{M}}^{\text {ncp }}(s)=S_{1} \alpha\left(x_{1}\right)+\cdots+S_{k} \alpha\left(x_{k}\right)$; similarly for $t$. We write $s \succeq_{\mathcal{M}}^{\text {ncp }} t$ if $[\alpha]_{\mathcal{M}}^{\text {ncp }}(s) \succeq[\alpha]_{\mathcal{M}}^{\text {ncp }}(t)$ holds for all assignments $\alpha$. Note that this condition can effectively be tested by requiring $S_{i} \geqslant T_{i}(1 \leqslant i \leqslant k)$.

Theorem 4.11. Let $\left(\mathcal{R}_{1} \cup \mathcal{R}_{2}\right) / \mathcal{S}$ be a relative $T R S, L$ a set of terminating terms, $\mathcal{M}$ a matrix interpretation with constant growth, $\mathcal{R}_{1} \subseteq \succeq_{\mathcal{M}}^{\text {ncp }}$, and $\mathcal{R}_{2} / \mathcal{S}$ be compatible with $\mathcal{M}$. Then $\mathrm{cp}_{L}\left(n, \rightarrow\left(\mathcal{R}_{1} \cup \mathcal{R}_{2}\right) / \mathcal{S}\right)=\mathcal{O}\left(\mathrm{cp}_{L}\left(n, \rightarrow_{\mathcal{R}_{1} /\left(\mathcal{R}_{2} \cup \mathcal{S}\right)}\right)+n\right)$. 
Proof. Throughout this proof we assume that $L=\mathcal{T}(\mathcal{F}, \mathcal{V})$. Since the matrix interpretation $\mathcal{M}$ has constant growth we have $\mathrm{cp}_{L}\left(n, \succ_{\mathcal{M}}\right)=\mathcal{O}(n)$. Since $\mathcal{R}_{2} / \mathcal{S}$ is compatible with the complexity pair $\left(\succ_{\mathcal{M}}, \succeq_{\mathcal{M}}\right)$ using Theorem 4.6 it remains to show that there is a constant $\Delta$ such that $u \rightarrow_{\mathcal{R}_{1}} v$ implies $\operatorname{dh}\left(u, \succ_{\mathcal{M}}\right)+\Delta \geqslant \operatorname{dh}\left(v, \succ_{\mathcal{M}}\right)$. Since $\mathcal{M}$ has constant growth there is a matrix $A$ such that $A \geqslant M_{1} \cdot \ldots \cdot M_{p}$ for any $p \in \mathbb{N}$ where the $M_{i}$ 's are matrices occurring in $\mathcal{M}$. Let $\delta=\max \left\{\vec{r} \mid l \rightarrow r \in \mathcal{R}_{1}\right\}$ (here $\vec{r}$ is the constant part of the interpretation of $r$ and max denotes the pointwise maximum of vectors). Note that $\delta$ is a vector.

Let $\Delta=(A \delta)_{11}$. Because the derivation height of a term $t$ with respect to $\succ_{\mathcal{M}}$ is determined by the first component of the vector $[\alpha]_{\mathcal{M}}(t)$ we have $\operatorname{dh}\left(u, \succ_{\mathcal{M}}\right)+\Delta \geqslant \operatorname{dh}\left(v, \succ_{\mathcal{M}}\right)$ whenever $[\alpha]_{\mathcal{M}}(u)+A \delta \geqslant[\alpha]_{\mathcal{M}}(v)$. To show the latter let $l \rightarrow r \in \mathcal{R}_{1}, u=C[l \sigma], v=C[r \sigma]$, $[\alpha]_{\mathcal{M}}(l)=L_{1} \alpha\left(x_{1}\right)+\cdots+L_{k} \alpha\left(x_{k}\right)+\vec{l}$, and $[\alpha]_{\mathcal{M}}(r)=R_{1} \alpha\left(x_{1}\right)+\cdots+R_{k} \alpha\left(x_{k}\right)+\vec{r}$.

By definition of $\delta$ we have $\vec{l}+\delta \geqslant \vec{r}$. Since $\mathcal{R}_{1} \subseteq \succeq_{\mathcal{M}}^{\text {ncp }}$ we have $L_{i} \geqslant R_{i}$ for all $1 \leqslant i \leqslant k$ and hence $L_{1} \alpha\left(x_{1}\right)+\cdots+L_{k} \alpha\left(x_{k}\right)+\vec{l}+\delta \geqslant R_{1} \alpha\left(x_{1}\right)+\cdots+R_{k} \alpha\left(x_{k}\right)+\vec{r}$ for any $\alpha$ and furthermore $L_{1} \alpha\left(x_{1} \sigma\right)+\cdots+L_{k} \alpha\left(x_{k} \sigma\right)+\vec{l}+\delta \geqslant R_{1} \alpha\left(x_{1} \sigma\right)+\cdots+R_{k} \alpha\left(x_{k} \sigma\right)+\vec{r}$ for any $\sigma$. The latter implies $D\left(L_{1} \alpha\left(x_{1} \sigma\right)+\cdots+L_{k} \alpha\left(x_{k} \sigma\right)+\vec{l}+\delta\right) \geqslant D\left(R_{1} \alpha\left(x_{1} \sigma\right)+\cdots+R_{k} \alpha\left(x_{k} \sigma\right)+\vec{r}\right)$ for any non-negative matrix $D$ and especially $D L_{1} \alpha\left(x_{1} \sigma\right)+\cdots+D L_{k} \alpha\left(x_{k} \sigma\right)+D \vec{l}+A \delta \geqslant$ $D R_{1} \alpha\left(x_{1} \sigma\right)+\cdots+D R_{k} \alpha\left(x_{k} \sigma\right)+D \vec{r}$ if $A \geqslant D$ (which is no restriction since $\mathcal{M}$ has constant growth and any $D$ that can occur is a matrix product of the shape $M_{1} \cdot \ldots \cdot M_{p} \leqslant A$ for some $p \in \mathbb{N})$. The proof concludes by the observation that the above inequation implies $[\alpha]_{\mathcal{M}}(C[l \sigma])+A \delta \geqslant[\alpha]_{\mathcal{M}}(C[r \sigma])$ for any context $C$.

We conclude this section with a discussion of the above theorem. Due to [21, Theorem 9] TMIs where each matrix $M$ satisfies $M_{(i, i)}<1$ for any $i \geqslant 2$ have constant growth. Since SLIs trivially adhere to this restriction Theorem 4.11 subsumes Corollary 4.7. The next example shows that this inclusion is strict.

Example 4.12. Let $\mathcal{R}_{1}=\{\mathrm{a}(x) \rightarrow \mathrm{c}(x)\}, \mathcal{R}_{2}=\{\mathrm{a}(\mathrm{b}(\mathrm{a}(x))) \rightarrow \mathrm{a}(\mathrm{b}(\mathrm{b}(\mathrm{a}(x))))\}$, and $\mathcal{S}=\varnothing$. Then the TMI $\mathcal{M}$ with

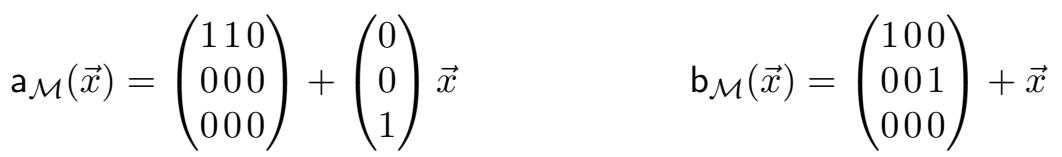

where $\mathrm{c}_{\mathcal{M}}(\vec{x})=\mathrm{a}_{\mathcal{M}}(\vec{x})$ has constant growth and transforms $\left(\mathcal{R}_{1} \cup \mathcal{R}_{2}\right) / \mathcal{S}$ into $\mathcal{R}_{1} /\left(\mathcal{R}_{2} \cup \mathcal{S}\right)$ according to Theorem 4.11. However, there exists no SLI that orients the rule in $\mathcal{R}_{2}$ strictly which shows that Corollary 4.7 cannot achieve this step.

\section{Relative Match-Bounds}

In this section we illustrate how the match-bound technique can be used to prove relative termination and estimate complexity bounds for relative rewriting. To maximize the power of the method we combine the ideas in [27] with the ones in [31. Preliminaries for matchbounds are introduced in Section 5.1. Section 5.2 shows how the technique works for linear systems before Section 5.3 extends applicability to non-left-linear systems. Automation is addressed in Section 5.4. Throughout this section we consider $L \subseteq \mathcal{T}(\mathcal{F})$ which does not affect the results by assuming that the signature $\mathcal{F}$ always contains a constant. 
5.1. Preliminaries. Let $\mathcal{F}$ be a signature, $\mathcal{R}$ a TRS over $\mathcal{F}$, and $L \subseteq \mathcal{T}(\mathcal{F})$ a set of ground terms. The set $\left\{t \in \mathcal{T}(\mathcal{F}) \mid s \rightarrow_{\mathcal{R}}^{*} t\right.$ for some $\left.s \in L\right\}$ of reducts of $L$ is denoted by $\rightarrow_{\mathcal{R}}^{*}(L)$. Given a set $N \subseteq \mathbb{N}$ of natural numbers, the signature $\mathcal{F} \times N$ is denoted by $\mathcal{F}_{N}$. Here function symbols $(f, c)$ with $f \in \mathcal{F}$ and $c \in N$ have the same arity as $f$ and are written as $f_{c}$. The mappings lift $t_{c}: \mathcal{F} \rightarrow \mathcal{F}_{\mathbb{N}}$, base: $\mathcal{F}_{\mathbb{N}} \rightarrow \mathcal{F}$, and height: $\mathcal{F}_{\mathbb{N}} \rightarrow \mathbb{N}$ are defined as $\operatorname{lift}_{c}(f)=f_{c}$, base $\left(f_{c}\right)=f$, and height $\left(f_{c}\right)=c$ for all $f \in \mathcal{F}$ and $c \in \mathbb{N}$. They are extended to terms, sets of terms, and TRSs in the obvious way. The TRS raise $(\mathcal{F})$ over the signature $\mathcal{F}_{\mathbb{N}}$ consists of all rules $f_{c}\left(x_{1}, \ldots, x_{n}\right) \rightarrow f_{c+1}\left(x_{1}, \ldots, x_{n}\right)$ with $f$ an $n$-ary function symbol in $\mathcal{F}, c \in \mathbb{N}$, and $x_{1}, \ldots, x_{n}$ pairwise distinct variables. The restriction of $\operatorname{raise}(\mathcal{F})$ to the signature $\mathcal{F}_{\{0, \ldots, c\}}$ is denoted by raise $(\mathcal{F})$. For terms $s, t \in \mathcal{T}\left(\mathcal{F}_{\mathbb{N}}, \mathcal{V}\right)$ we write $s \uparrow t$ for the least term $u$ with $s \rightarrow_{\text {raise }(\mathcal{F})}^{*} u$ and $t \rightarrow_{\text {raise }(\mathcal{F})}^{*} u$. Here least refers to the (sum of the) lengths of the joining sequences. We extend this notion to $\uparrow S$ for finite non-empty sets $S \subseteq \mathcal{T}\left(\mathcal{F}_{\mathbb{N}}, \mathcal{V}\right)$ in the obvious way. Note that $\uparrow S$ is undefined whenever $S$ contains two terms $s$ and $t$ such that base $(s) \neq$ base $(t)$. The TRS match $(\mathcal{R})$ over the signature $\mathcal{F}_{\mathbb{N}}$ consists of all rewrite rules $l^{\prime} \rightarrow \operatorname{lift}_{c}(r)$ for which there exists a rule $l \rightarrow r \in \mathcal{R}$ such that base $\left(l^{\prime}\right)=l$ and $c=1+\min \left\{\right.$ height $\left.\left(l^{\prime}(p)\right) \mid p \in \operatorname{Pos}_{\mathcal{F}}(l)\right\}$. Here $c \in \mathbb{N}$. The restriction of match $(\mathcal{R})$ to the signature $\mathcal{F}_{\{0, \ldots, c\}}$ is denoted by $\operatorname{match}_{c}(\mathcal{R})$. To be able to apply the matchbound technique to non-left-linear TRSs we define the relation $r_{\text {match }(\mathcal{R})}$ on $\mathcal{T}\left(\mathcal{F}_{\mathbb{N}}, \mathcal{V}\right)$ as follows: $s \stackrel{r}{\rightarrow}_{\operatorname{match}(\mathcal{R})} t$ if and only if there exist a rewrite rule $l \rightarrow r \in$ match $(\mathcal{R})$, a position $p \in \operatorname{Pos}(s)$, a context $C$, and terms $s_{1}, \ldots, s_{n}$ such that $l=C\left[x_{1}, \ldots, x_{n}\right]$ with all variables displayed, $\left.s\right|_{p}=C\left[s_{1}, \ldots, s_{n}\right]$, base $\left(s_{i}\right)=\operatorname{base}\left(s_{j}\right)$ whenever $x_{i}=x_{j}$ for all $i, j \in\{1, \ldots, n\}$, and $t=s[r \sigma]_{p}$. Here the substitution $\sigma$ is defined as follows:

$$
\sigma(x)= \begin{cases}\uparrow\left\{s_{i} \mid x_{i}=x \text { with } i \in\{1, \ldots, n\}\right\} & \text { if } x \in\left\{x_{1}, \ldots, x_{n}\right\} \\ x & \text { otherwise }\end{cases}
$$

Let $L$ be a set of ground terms. A TRS $\mathcal{R}$ is called match-bounded for $L$ if there exists a $c \in \mathbb{N}$ such that the maximum height of function symbols occurring in terms in $\rightarrow_{\operatorname{match}(\mathcal{R})}^{*}\left(\operatorname{lift}_{0}(L)\right)$ is at most $c$. Similarly, a TRS $\mathcal{R}$ is called match-raise-bounded for $L$ if there exists a $c \in \mathbb{N}$ such that the maximum height of function symbols occurring in terms belonging to $\stackrel{r}{\rightarrow}_{\text {match }(\mathcal{R})}^{*}\left(\operatorname{lift}_{0}(L)\right)$ is at most $c$. If we want to make the bound $c$ precise, we say that $\mathcal{R}$ is match(-raise)-bounded for $L$ by $c$. If we do not specify the set of terms $L$ then it is assumed that $L=\mathcal{T}(\mathcal{F})$. The main result underlying the match-bound technique states that a TRS $\mathcal{R}$ is terminating for a language $L$ if $\mathcal{R}$ is linear and match-bounded for $L$ or $\mathcal{R}$ is non-duplicating and match-raise-bounded for $L$.

In order to prove that a TRS $\mathcal{R}$ is match(-raise)-bounded for some language $L$, the idea is to construct a (quasi-deterministic and raise-consistent) tree automaton that is compatible with match $(\mathcal{R})$ and $\operatorname{lift}_{0}(L)$. In the following we briefly recall the most important definitions in this connection. A tree automaton $\mathcal{A}=\left(\mathcal{F}, Q, Q_{f}, \Delta\right)$ consists of a signature $\mathcal{F}$, a finite set of states $Q$, a set of final states $Q_{f} \subseteq Q$, and a set of transitions $\Delta$ of the form $f\left(q_{1}, \ldots, q_{n}\right) \rightarrow q$ or $p \rightarrow q$ where $f$ is an $n$-ary function symbol in $\mathcal{F}$ and $p, q, q_{1}, \ldots, q_{n} \in Q$. The language $\mathcal{L}(\mathcal{A})$ of $\mathcal{A}$ is the set of ground terms $t \in \mathcal{T}(\mathcal{F})$ such that $t \rightarrow_{\Delta}^{*} q$ for some $q \in Q_{f}$. We say that $\mathcal{A}$ is compatible with a TRS $\mathcal{R}$ and a language $L$ if $L \subseteq \mathcal{L}(\mathcal{A})$ and for each rewrite rule $l \rightarrow r \in \mathcal{R}$ and state substitution $\sigma: \operatorname{Var}(l) \rightarrow Q$ such that $l \sigma \rightarrow_{\Delta}^{*} q$ it holds that $r \sigma \rightarrow_{\Delta}^{*} q$. For left-linear $\mathcal{R}$ it is known that $\rightarrow_{\mathcal{R}}^{*}(L) \subseteq \mathcal{L}(\mathcal{A})$ whenever $\mathcal{A}$ is compatible with $\mathcal{R}$ and $L$ [7]. To obtain a similar result for non-left-linear TRSs, in [17] quasi-deterministic automata are introduced. Let $\mathcal{A}=\left(\mathcal{F}, Q, Q_{f}, \Delta\right)$ be a 
tree automaton. We say that a state $p$ subsumes a state $q$ if $p$ is final when $q$ is final and for all transitions $f\left(u_{1}, \ldots, q, \ldots, u_{n}\right) \rightarrow u \in \Delta$, the transition $f\left(u_{1}, \ldots, p, \ldots, u_{n}\right) \rightarrow u$ belongs to $\Delta$. For a left-hand side $l \in \operatorname{lhs}(\Delta)$ of a transition, the set $\{q \mid l \rightarrow q \in \Delta\}$ of possible right-hand sides is denoted by $Q(l)$. The automaton $\mathcal{A}$ is said to be quasideterministic if for every $l \in \operatorname{lhs}(\Delta)$ there exists a state $p \in Q(l)$ which subsumes every other state in $Q(l)$. In general, $Q(l)$ may contain more than one state that satisfies the above property. In the following we assume that there is a unique designated state in $Q(l)$, which we denote by $p_{l}$. The set of all designated states is denoted by $Q_{d}$ and the restriction of $\Delta$ to transitions $l \rightarrow q$ that satisfy $q=p_{l}$ is denoted by $\Delta_{d}$. In [17] it is shown that the tree automaton induced by $\Delta_{d}$ is deterministic and accepts the same language as $\mathcal{A}$. For non-left-linear TRSs $\mathcal{R}$ we modify the above definition of compatibility by demanding that the tree automaton $\mathcal{A}$ is quasi-deterministic and for each rewrite rule $l \rightarrow r \in \mathcal{R}$ and state substitution $\sigma: \operatorname{Var}(l) \rightarrow Q_{d}$ with $l \sigma \rightarrow_{\Delta_{d}}^{*} q$ it holds that $r \sigma \rightarrow_{\Delta}^{*} q$. To ensure that quasideterministic and compatible tree automata can be used to prove match-raise-boundedness of a TRS $\mathcal{R}$ it must be guaranteed that the obtained tree automata are closed under the implicit raise-steps caused by the relation $\stackrel{r}{\rightarrow}_{\operatorname{match}(\mathcal{R})}$. To this end we additionally require that the resulting tree automata fulfill the property defined below. Let $\mathcal{A}=\left(\mathcal{F}_{N}, Q, Q_{f}, \Delta\right)$ be a tree automaton with $N$ a finite subset of $\mathbb{N}$. We say that $\mathcal{A}$ is raise-consistent if for every transition $f_{c}\left(q_{1}, \ldots, q_{n}\right) \rightarrow q \in \Delta$ and left-hand side $f_{d}\left(q_{1}, \ldots, q_{n}\right) \in \operatorname{Ihs}(\Delta)$ with $c<_{\mathbb{N}} d$, the transition $f_{d}\left(q_{1}, \ldots, q_{n}\right) \rightarrow q$ belongs to $\Delta$.

By a remark in [8] we know that the derivation height of a term in $L$ is at most linear in the size of the term whenever $\mathcal{R}$ is match-bounded for $L$. It is easy to extend this result to match-raise-boundedness and hence to non-duplicating TRSs. To this end we need the following notions. Let $\mathcal{M u l}(\mathbb{N})$ denote the set of all finite multisets over $\mathbb{N}$. For any $M \in \mathcal{M u l}(\mathbb{N})$ we write $M(n)$ to denote how often the number $n \in \mathbb{N}$ occurs in $M$. Let $M, N \in \mathcal{M u l}(\mathbb{N})$ be two multisets. We write $M \cup N$ for the multiset sum of $M$ and $N$ where $(M \cup N)(n)=M(n)+N(n)$ for all $n \in \mathbb{N}$ and $M \subseteq N$ for the multiset inclusion, i.e., $M(n) \leqslant N(n)$ for all $n \in \mathbb{N}$. The multiset difference $M \backslash N$ is defined as $(M \backslash N)(n)=M(n)-N(n)$ if $M(n)>N(n)$ and $(M \backslash N)(n)=0$ otherwise, for all $n \in \mathbb{N}$. We write $M \succ_{\text {mul }} N$ if there are multisets $X$ and $Y$ such that $N=(M \backslash X) \cup Y, X \neq \varnothing$, and for all $m \in Y$ there is an $n \in X$ such that $n<_{\mathbb{N}} m$. We write $M \succeq_{\text {mul }} N$ if $M \succ_{\text {mul }} N$ or $M=N$. Let $\mathcal{F}$ be some signature. We extend the orderings $\succ_{\text {mul }}$ and $\succeq_{\text {mul }}$ to terms over the signature $\mathcal{F}_{\mathbb{N}}$ as follows: we have $s \succ_{\text {mul }} t$ if $\mathcal{H}(s) \succ_{\text {mul }} \mathcal{H}(t)$ and $s \succeq_{\text {mul }} t$ if $\mathcal{H}(s) \succeq_{\text {mul }} \mathcal{H}(t)$ for terms $s, t \in \mathcal{T}\left(\mathcal{F}_{\mathbb{N}}, \mathcal{V}\right)$. Here $\mathcal{H}(t)=\left\{\right.$ height $\left.(t(p)) \mid p \in \operatorname{Pos}_{\mathcal{F}}(t)\right\}$ denotes the multiset of the heights of function symbols occurring in the term $t$.

Theorem 5.1. Let $\mathcal{R}$ be a TRS and $L$ be a language. If $\mathcal{R}$ is linear and match-bounded or non-duplicating and match-raise-bounded for $L$ then $\mathrm{cp}_{L}\left(n, \rightarrow_{\mathcal{R}}\right)=\mathcal{O}(n)$.

Proof. Assume that $\mathcal{R}$ is match-raise-bounded for $L$ and hence terminating on $L$. (Note that for a linear TRS $\mathcal{R}$, match-boundedness coincides with match-raise-boundedness.) Let

$$
t \rightarrow_{\mathcal{R}} t_{1} \rightarrow_{\mathcal{R}} \cdots \rightarrow_{\mathcal{R}} t_{m-1} \rightarrow_{\mathcal{R}} t_{m}
$$

be an arbitrary (terminating) rewrite sequence with $t \in L$. Since every $\rightarrow_{\mathcal{R}}$ rewrite sequence can be lifted to a $\stackrel{\mathrm{r}}{\rightarrow}_{\text {match }(\mathcal{R})}$ rewrite sequence [18, Lemma 12] we obtain a derivation

$$
t^{\prime} \stackrel{r}{\rightarrow}_{\operatorname{match}(\mathcal{R})} t_{1}^{\prime} \stackrel{r}{\rightarrow}_{\operatorname{match}(\mathcal{R})} \cdots \stackrel{r}{\rightarrow}_{\operatorname{match}(\mathcal{R})} t_{m-1}^{\prime} \stackrel{r}{\rightarrow}_{\operatorname{match}(\mathcal{R})} t_{m}^{\prime}
$$

such that $t^{\prime}=\operatorname{lift}_{0}(t)$ and base $\left(t_{i}^{\prime}\right)=t_{i}$ for all $i \in\{1, \ldots, m\}$. From the proof of [18, Lemma 8] we know that for any non-duplicating TRS $\mathcal{R}$ we have $\stackrel{r}{\rightarrow}_{\text {match }(\mathcal{R})} \subseteq \succ_{\text {mul }}$. It 
follows that $t_{i}^{\prime} \succ_{\text {mul }} t_{i+1}^{\prime}$ for all $i \in\{0, \ldots, m-1\}$. Here $t_{0}^{\prime}=t^{\prime}$. Since $\mathcal{R}$ is match-raisebounded for $L$, all terms in this latter sequence belong to $\mathcal{T}\left(\mathcal{F}_{\{0, \ldots, c\}}\right)$ for some $c \in \mathbb{N}$. Let $k$ be the maximal number of function symbols occurring in some right-hand side in $\mathcal{R}$. Due to a remark in [5] we know that the length of the $\succ_{\text {mul }}$ chain from $t^{\prime}$ to $t_{m}^{\prime}$ is bounded by $\left\|t^{\prime}\right\| \cdot(k+1)^{c}$. Since $\left\|t^{\prime}\right\|=\|t\|$ and the $\succ_{\text {mul }}$ chain starting at $t^{\prime}$ is at least as long as the lifted and hence original rewrite sequence, we conclude that the length of the $\mathcal{R}$-rewrite sequence starting at the term $t$ is bounded by $\|t\| \cdot(k+1)^{c}$.

Based on Theorem 5.1 it is easy to use the match-bound technique to estimate the complexity of a relative TRS $\mathcal{R} / \mathcal{S}$; just check for match(-raise)-boundedness of $\mathcal{R} \cup \mathcal{S}$. This process either succeeds by proving that the combined TRS is match(-raise)-bounded, or, when $\mathcal{R} \cup \mathcal{S}$ cannot be proved to be match(-raise)-bounded, it fails. Since the construction of a (quasi-deterministic, raise-consistent, and) compatible tree automaton does not terminate for TRSs that are not match(-raise)-bounded, the latter situation typically does not happen. This behavior causes a serious problem since we cannot benefit from relative rewriting, i.e., $\mathcal{R} / \mathcal{S}$ is match(-raise)-bounded if and only if $\mathcal{R} \cup \mathcal{S}$ is. In [27] this problem has been addressed by specifying an upper bound on the heights that can be introduced by rewrite rules in match $(\mathcal{S})$. So one tries to find a $c \in \mathbb{N}$ such that the maximum height of function symbols occurring in reductions with the TRS match $\operatorname{Tat}_{c+1}(\mathcal{R}) \cup \operatorname{match}_{c}(\mathcal{S}) \cup \operatorname{lift}_{c}(\mathcal{S})$ is at most $c$. If such a bound can be established we know that $\mathcal{R} / \mathcal{S}$ is terminating and in addition that it admits at most linear complexity. In the following we extend this approach to better suit relative rewriting. To this end we introduce a new enrichment match- $\mathrm{RT}^{c}(\mathcal{R} / \mathcal{S})$ where the rewrite rules in match- $\mathrm{RT}^{c}(\mathcal{R} / \mathcal{S})$ which originate from size-preserving or size-decreasing rules in $\mathcal{S}$ are labeled in such a way that they do not increase the heights of the function symbols in a contracted redex.

To simplify the presentation we first consider linear TRSs only. The extension to nonduplicating TRSs is explained in Section 5.3.

5.2. RT-Bounds for Left-Linear Relative TRSs. As proposed in [27] we design the new enrichment match- $\mathrm{RT}^{c}(\mathcal{R} / \mathcal{S})$ such that rules originating from $\mathcal{S}$ may introduce function symbols with height at most $c$. In addition (as in [31]) we try to keep the heights of the function symbols in a contracted redex if a size-preserving or size-decreasing rewrite rule in $\mathcal{S}$ (after dropping all heights) is applied.

Definition 5.2. Let $\mathcal{S}$ be a TRS over a signature $\mathcal{F}$ and $c \in \mathbb{N}$. The TRS match-RT ${ }^{c}(\mathcal{S})$ over the signature $\mathcal{F}_{\mathbb{N}}$ consists of all rules $l^{\prime} \rightarrow \operatorname{lift}_{d}(r)$ such that base $\left(l^{\prime}\right) \rightarrow r \in \mathcal{S}$ and

$$
d= \begin{cases}\min \left\{c, \text { height }\left(l^{\prime}(\epsilon)\right)\right\} & \text { if } \| \text { base }\left(l^{\prime}\right)\|\geqslant\| r \| \text { and } \\ \min \left\{c, 1+\operatorname{height}\left(l^{\prime}(p)\right) \mid p \in \operatorname{Pos}_{\mathcal{F}}\left(l^{\prime}\right)\right\} & \text { lift } \\ \text { otheight }\left(l^{\prime}(\epsilon)\right) & \text { base } \left.\left(l^{\prime}\right)\right)=l^{\prime}\end{cases}
$$

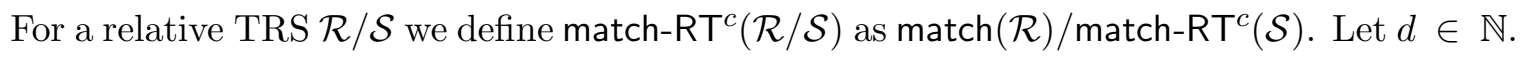

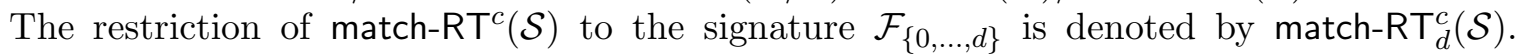
Likewise the relative TRS match- $\mathrm{RT}_{d}^{c}(\mathcal{R} / \mathcal{S})$ is defined as match $(\mathcal{R}) / \operatorname{match}-\mathrm{RT}_{d}^{c}(\mathcal{S})$. In case $c=d$ then match-RT ${ }_{d}^{c}(\mathcal{R} / \mathcal{S})$ is abbreviated by match-RT ${ }_{c}(\mathcal{R} / \mathcal{S})$ and match- $\mathrm{RT}_{d}^{c}(\mathcal{S})=$ match-RT $T_{c}(\mathcal{S})$. 
The idea behind the requirement $\|$ base $\left(l^{\prime}\right)\|\geqslant\| r \|$ in the above definition is that such rules cannot yield an increase with respect to the multiset measure of heights. Let us illustrate the above definition on an example.

Example 5.3. Consider the relative TRS $\mathcal{R} / \mathcal{S}$ with $\mathcal{R}$ consisting of the rewrite rule

$$
1: \operatorname{rev}(x) \rightarrow \operatorname{rev}^{\prime}(x, \operatorname{nil})
$$

and $\mathcal{S}$ consisting of the rewrite rules

$$
2: \operatorname{rev}^{\prime}(\operatorname{nil}, y) \rightarrow y \quad 3: \operatorname{rev}^{\prime}(\operatorname{cons}(x, y), z) \rightarrow \operatorname{rev}^{\prime}(y, \operatorname{cons}(x, z))
$$

Then the rewrite rules

$$
\begin{array}{cc}
\operatorname{rev}_{0}(x) \rightarrow \operatorname{rev}_{1}^{\prime}\left(x, \operatorname{nil}_{1}\right) & \operatorname{rev}_{1}(x) \rightarrow \operatorname{rev}_{2}^{\prime}\left(x, \operatorname{nil}_{2}\right) \\
\operatorname{rev}_{2}(x) \rightarrow \operatorname{rev}_{3}^{\prime}\left(x, \operatorname{nil}_{3}\right) & \cdots
\end{array}
$$

belong to $\operatorname{match}(\mathcal{R})$ and match- $\mathrm{RT}^{1}(\mathcal{S})$ contains the rules

$$
\begin{array}{cl}
\operatorname{rev}_{0}^{\prime}\left(\operatorname{nil}_{0}, y\right) \rightarrow y & \operatorname{rev}_{0}^{\prime}\left(\operatorname{cons}_{0}(x, y), z\right) \rightarrow \operatorname{rev}_{0}^{\prime}\left(y, \operatorname{cons}_{0}(x, z)\right) \\
\operatorname{rev}_{0}^{\prime}\left(\operatorname{nil}_{1}, y\right) \rightarrow y & \operatorname{rev}_{0}^{\prime}\left(\operatorname{cons}_{1}(x, y), z\right) \rightarrow \operatorname{rev}_{1}^{\prime}\left(y, \operatorname{cons}_{1}(x, z)\right) \\
\ldots & \operatorname{rev}_{2}^{\prime}\left(\operatorname{cons}_{1}(x, y), z\right) \rightarrow \operatorname{rev}_{1}^{\prime}\left(y, \operatorname{cons}_{1}(x, z)\right)
\end{array}
$$

Both TRSs together constitute match- $\mathrm{RT}^{1}(\mathcal{R} / \mathcal{S})$.

The new enrichment match- $\mathrm{RT}^{c}(\mathcal{R} / \mathcal{S})$ allows to prove the complexity of the rewrite rules in $\mathcal{R}$ relative to the rules in $\mathcal{S}$.

Definition 5.4. Let $\mathcal{R} / \mathcal{S}$ be a relative TRS. We call $\mathcal{R} / \mathcal{S}$ match-RT-bounded for a language $L$ if there exists a $c \in \mathbb{N}$ such that the height of function symbols occurring in terms in $\rightarrow_{\text {match-RT }}^{*}(\mathcal{R} / \mathcal{S})\left(\operatorname{lift}_{0}(L)\right)$ is at most $c$.

An immediate consequence of the next lemma is that every derivation in $\mathcal{R} / \mathcal{S}$ can be lifted to a match- $\mathrm{RT}^{c}(\mathcal{R} / \mathcal{S})$-sequence of the same length. This result is used later on to infer termination and complexity results for relative rewriting.

Lemma 5.5. Let $\mathcal{R} / \mathcal{S}$ be a left-linear relative $T R S$ and $c \in \mathbb{N}$. If $u \rightarrow_{\mathcal{R}} v\left(u \rightarrow_{\mathcal{S}} v\right)$ then for all terms $u^{\prime}$ with base $\left(u^{\prime}\right)=u$ there exists a term $v^{\prime}$ such that base $\left(v^{\prime}\right)=v$ and $u^{\prime} \rightarrow_{\operatorname{match}(\mathcal{R})} v^{\prime}\left(u^{\prime} \rightarrow_{\text {match-RT }}(\mathcal{S}) v^{\prime}\right)$.

Proof. Straightforward.

To be able to prove that a relative TRS $\mathcal{R} / \mathcal{S}$ admits a linear upper complexity bound whenever it is match-RT-bounded for a language $L$ we slightly modify the orderings $\succ_{\text {mul }}$ and $\succeq_{\text {mul }}$. Let $M, N \in \mathcal{M u l}(\mathbb{N})$ be multisets. The function $\operatorname{drop}_{n}(M)$ removes all occurrences of the number $n \in \mathbb{N}$ from $M$. So for all $m \in \mathbb{N}$ we have $\operatorname{drop}_{n}(M)(m)=0$ if $m=n$ and $\operatorname{drop}_{n}(M)(m)=M(m)$ otherwise. The orderings $\succ_{\text {mul }}^{c}$ and $\succeq_{\text {mul }}^{c}$ are defined as $M \succ_{\text {mul }}^{c} N$ if $\operatorname{drop}_{c}(M) \succ_{\text {mul }} \operatorname{drop}_{c}(N)$ and $M \succeq_{\text {mul }}^{c} N$ if $\operatorname{drop}_{c}(M) \succeq_{\text {mul }} \operatorname{drop}_{c}(N)$. Let $\mathcal{F}$ be some signature. We extend $\succ_{\text {mul }}^{c}$ and $\succeq_{\text {mul }}^{c}$ to terms over the signature $\mathcal{F}_{\mathbb{N}}$ as follows: we have $s \succ_{\text {mul }}^{c} t$ if $\mathcal{H}(s) \succ_{\text {mul }}^{c} \mathcal{H}(t)$ and $s \succeq_{\text {mul }}^{c} t$ if $\mathcal{H}(s) \succeq_{\text {mul }}^{c} \mathcal{H}(t)$ for terms $s, t \in \mathcal{T}\left(\mathcal{F}_{\mathbb{N}}, \mathcal{V}\right)$. The basic idea behind the new orderings $\succ_{\text {mul }}^{c}$ and $\succeq_{\text {mul }}^{c}$ is that rewrite rules in match- $\operatorname{RT}_{c}(\mathcal{R} / \mathcal{S})$ which originate from $\mathcal{R}$ are compatible with $\succ_{\text {mul }}^{c}$ and the rules originating from $\mathcal{S}$ are compatible with $\succeq_{\text {mul }}^{c}$. However there is one problem. If $\mathcal{R}$ contains a collapsing rule $l \rightarrow r$ 
then the rule $\operatorname{lift}_{c}(l) \rightarrow \operatorname{lift}_{c}(r)$ appears in match-RT $(\mathcal{R} / \mathcal{S})$ which cannot be oriented via the ordering $\succ_{\text {mul }}^{c}$ although $\operatorname{lift}_{c}(l) \succ_{\text {mul }} \operatorname{lift}_{c}(r)$. The problem is that collapsing rewrite rules do not increase the heights of function symbols in a contracted redex because the right-hand sides consist just of single variables. To avoid this problem we assume in the following that $\mathcal{R}$ is non-collapsing. For collapsing $\mathcal{R}$ one could follow the approach in [31] which can handle collapsing rewrite rules because it does not not use an upper bound $c$ to limit the heights that can be introduced by the enriched system. However, a disadvantages of this approach is that the heights of a contracted redex are increased more often. So, apart from the collapsing case the approach presented here is more powerful than the one introduced in [31] and completely subsumes the approach in [27].

Lemma 5.6. Let $\mathcal{R}$ and $\mathcal{S}$ be two non-duplicating TRSs and $c \in \mathbb{N}$. If $\mathcal{R}$ is non-collapsing then $\rightarrow_{\text {match }_{c}(\mathcal{R})} \subseteq \succ_{\text {mul }}^{c}$ and $\rightarrow_{\text {match }-\mathrm{RT}}(\mathcal{S}) \subseteq \succeq_{\text {mul }}^{c}$.

Proof. From the proof of [8, Lemma 17] we know that for a non-duplicating TRS $\mathcal{R}$ and terms $s$ and $t$ such that $s \rightarrow_{\text {match }_{c}(\mathcal{R})} t$ we have $s \succ_{\text {mul }} t$. So there are multisets $X$ and $Y$ such that $\mathcal{H}(t)=(\mathcal{H}(s) \backslash X) \cup Y, X \neq \varnothing$, and for all $d^{\prime} \in Y$ there is a $d \in X$ such that $d<_{\mathbb{N}} d^{\prime}$. Because $\mathcal{R}$ is non-collapsing we know from the definition of $\operatorname{match}_{c}(\mathcal{R})$ that there is a $d \in X$ such that $d<_{\mathbb{N}} c$ and $d<_{\mathbb{N}} d^{\prime}$ for all $d^{\prime} \in Y$. From this it follows that $\operatorname{drop}_{c}(\mathcal{H}(t))=\left(\operatorname{drop}_{c}(\mathcal{H}(s)) \backslash \operatorname{drop}_{c}(X)\right) \cup \operatorname{drop}_{c}(Y)$, $\operatorname{drop}_{c}(X) \neq \varnothing$, and for all $d^{\prime} \in$ $\operatorname{drop}_{c}(Y)$ there is a $d \in \operatorname{drop}_{c}(X)$ such that $d<_{\mathbb{N}} d^{\prime}$. As an immediate consequence we have $\operatorname{drop}_{c}(\mathcal{H}(s)) \succ_{\text {mul }} \operatorname{drop}_{c}(\mathcal{H}(t))$ and hence $s \succ_{\text {mul }}^{c} t$.

Now let $s$ and $t$ be terms and $l \rightarrow r$ be a rewrite rule in match-RT $T_{c}(\mathcal{S})$ such that $s \rightarrow_{\{l \rightarrow r\}} t$. According to Definition 5.2 we have to consider two cases. The first case amounts to $\|l\| \geqslant\|r\|$ where all function symbols in $l$ and $r$ have the same heights. But then non-duplication of $\mathcal{S}$ implies $\mathcal{H}(s) \supseteq \mathcal{H}(t)$ and thus $s \succeq_{\text {mul }}^{c} t$. In the other case if $l \rightarrow r$ is non-collapsing and $l \notin \operatorname{lift}_{c}$ (base $\left.(l)\right)$ then we obtain $s \succ_{\text {mul }}^{c} t$ as before and hence also $s \succeq_{\text {mul }}^{c} t$. If $l \in \operatorname{lift}_{c}(\operatorname{base}(l))$ then $\operatorname{drop}_{c}(\mathcal{H}(s)) \supseteq \operatorname{drop}_{c}(\mathcal{H}(t))$ since $\operatorname{drop}_{c}(\mathcal{H}(l))=\operatorname{drop}_{c}(\mathcal{H}(r))=\varnothing$ and if $l \rightarrow r$ is collapsing then $\mathcal{H}(s) \supseteq \mathcal{H}(t)$ since $\mathcal{H}(r)=\varnothing$. Hence in both situations $s \succeq_{\text {mul }}^{c} t$.

Since the length of every $\succ_{\text {mul }}^{c}$ chain is bounded by a function linear in the size of the starting term - if the size-increase of the terms in the chain can be bounded by a constantwe can prove that the complexity induced by the relative TRS $\mathcal{R} / \mathcal{S}$ on some language $L$ is at most linear if $\mathcal{R} / \mathcal{S}$ is match-RT-bounded for $L$.

Theorem 5.7. Let $\mathcal{R} / \mathcal{S}$ be a linear relative $T R S$ and $\mathcal{R}$ be non-collapsing. If $\mathcal{R} / \mathcal{S}$ is match$R T$-bounded for a language $L$ then $\mathcal{R} / \mathcal{S}$ is terminating on $L$ and $\mathrm{cp}_{L}\left(n, \rightarrow_{\mathcal{R} / \mathcal{S}}\right)=\mathcal{O}(n)$.

Proof. First we show that $\mathcal{R} / \mathcal{S}$ is terminating on $L$. Assume to the contrary that there is an infinite rewrite sequence of the form

$$
t_{1} \rightarrow_{\mathcal{R} / \mathcal{S}} t_{2} \rightarrow_{\mathcal{R} / \mathcal{S}} t_{3} \rightarrow_{\mathcal{R} / \mathcal{S}} \cdots
$$

with $t_{1} \in L$. Because $\mathcal{R} \cup \mathcal{S}$ is left-linear and $\mathcal{R} / \mathcal{S}$ is match-RT-bounded for $L$ by a $c \in \mathbb{N}$, according to Lemma 5.5, the above derivation can be lifted to an infinite match- $\operatorname{RT}^{c}(\mathcal{R} / \mathcal{S})$ rewrite sequence

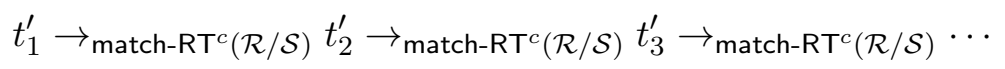

starting from $t_{1}^{\prime}=\operatorname{lift}_{0}\left(t_{1}\right)$ such that base $\left(t_{i}^{\prime}\right)=t_{i}$ for all $i \geqslant 1$ and the height of every function symbol occurring in a term in the lifted sequence is at most $c$. Hence the employed 
rewrite rules in the derivation emanating from $t_{1}^{\prime}$ must come from match-R $\mathrm{T}_{c}(\mathcal{R} / \mathcal{S})$. With help of Lemma 5.6. transitivity of $\succeq_{\text {mul }}^{c}$, and compatibility of the orderings $\succ_{\text {mul }}^{c}$ and $\succeq_{\text {mul }}^{c}$ we deduce that $t_{i}^{\prime} \succ_{\text {mul }}^{c} t_{i+1}^{\prime}$ for all $i \geqslant 1$. However, this is excluded because $<_{\mathbb{N}}$ is well-founded on $\{0, \ldots, c\}$ and hence $\succ_{\text {mul }}^{c}$ is well-founded on $\mathcal{T}\left(\mathcal{F}_{\{0, \ldots, c\}}, \mathcal{V}\right)$.

To prove the second part of the theorem, consider an arbitrary (terminating) rewrite sequence

$$
u \rightarrow_{\mathcal{R} / \mathcal{S}} u_{1} \rightarrow_{\mathcal{R} / \mathcal{S}} \cdots \rightarrow_{\mathcal{R} / \mathcal{S}} u_{m}
$$

with $u \in L$. Similar as before this rewrite sequence can be lifted to a match- $\operatorname{RT}^{c}(\mathcal{R} / \mathcal{S})$ sequence of the same length

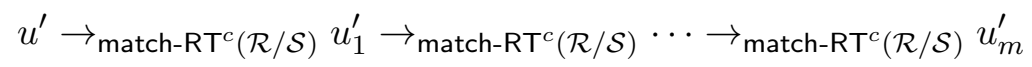

such that $u^{\prime}=\operatorname{lift}_{0}(u)$ and $u_{i}^{\prime} \succ_{\text {mul }}^{c} u_{i+1}^{\prime}$ for all $i \in\{0, \ldots, m-1\}$. Here $u_{0}^{\prime}=u^{\prime}$ and $c \in \mathbb{N}$ such that the relative TRS $\mathcal{R} / \mathcal{S}$ is match-RT-bounded for $L$ by $c$. Similar as in the proof of Theorem 5.1 we can conclude that the length of the $\mathcal{R} / \mathcal{S}$-rewrite sequence starting at the term $u$ is bounded by $\|u\| \cdot(k+1)^{c}$ where $k$ is the maximal number of function symbols occurring in some right-hand side in $\mathcal{R} \cup \mathcal{S}$; just replace $\succ_{\text {mul }}$ by $\succ_{\text {mul }}^{c}$.

We conclude this subsection with an example.

Example 5.8. The relative TRS $\mathcal{R} / \mathcal{S}$ of Example 5.3 is match-RT-bounded for $\mathcal{T}(\mathcal{F})$ by 1 . Here $\mathcal{F}=\left\{\right.$ nil, cons, rev, $\left.\operatorname{rev}^{\prime}\right\}$. Due to Theorem 5.7 we can conclude that $\mathcal{R} / \mathcal{S}$ admits at most linear derivational complexity. In Section 5.4 it is explained how match-RT-boundedness can be checked automatically.

5.3. Raise-RT-Bounds for Non-Left-Linear Relative TRSs. In order to generalize Theorem 5.7 to non-duplicating relative TRSs we consider the relation $\stackrel{r}{\rightarrow}_{\text {match-RT }}(\mathcal{R} / \mathcal{S})$ instead of $\rightarrow_{\text {match-RT }}{ }^{c}(\mathcal{R} / \mathcal{S})$ which uses raise-rules to deal with non-left-linearity. Thereby

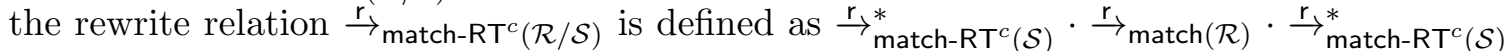
where $\stackrel{r}{\rightarrow}_{\text {match-RTc }}(\mathcal{S})$ is defined similar to $\stackrel{r}{\rightarrow}_{\text {match }(\mathcal{R})}$ (but based on match-RT ${ }^{c}(\mathcal{S})$ instead of match $(\mathcal{R})$ ). This is essential to lift rewrite sequences in the relative TRS $\mathcal{R} / \mathcal{S}$ to sequences in match- $\mathrm{RT}^{c}(\mathcal{R} / \mathcal{S})$.

Definition 5.9. Let $\mathcal{R} / \mathcal{S}$ be a relative TRS. We call $\mathcal{R} / \mathcal{S}$ match-raise-RT-bounded for a language $L$ if there exists a number $c \in \mathbb{N}$ such that the height of function symbols occurring in terms belonging to $\stackrel{\mathrm{r}}{\rightarrow}_{\text {match-RT}}^{*}(\mathcal{R} / \mathcal{S})\left(\operatorname{lift}_{0}(L)\right)$ is at most $c$.

Note that for left-linear relative TRSs, match-raise-RT-boundedness coincides with match-RT-boundedness. By using the relation $\stackrel{r}{\rightarrow}_{\text {match-RT }}{ }^{c}(\mathcal{R} / \mathcal{S})$ every derivation induced by the relative TRS $\mathcal{R} / \mathcal{S}$ can be simulated via the rewrite rules in match- $\operatorname{RT}^{c}(\mathcal{R} / \mathcal{S})$.

Lemma 5.10. Let $\mathcal{R} / \mathcal{S}$ be a relative TRS and $c \in \mathbb{N}$. If $u \rightarrow_{\mathcal{R}} v\left(u \rightarrow_{\mathcal{S}} v\right)$ then for all terms $u^{\prime}$ with base $\left(u^{\prime}\right)=u$ there exists a term $v^{\prime}$ such that base $\left(v^{\prime}\right)=v$ and $u^{\prime} \stackrel{\mathrm{r}}{\rightarrow}_{\operatorname{match}(\mathcal{R})} v^{\prime}$ $\left(u^{\prime} \stackrel{\mathrm{r}}{\rightarrow}_{\text {match-RT }}{ }^{c}(\mathcal{S}) v^{\prime}\right)$.

Proof. Straightforward. 
Before we can prove that match-raise-RT-boundedness of $\mathcal{R} / \mathcal{S}$ induces a linear upper bound on the complexity we have to ensure that the raise-rules implicitly used by the relation $\stackrel{r}{\rightarrow}_{\text {match- } R T^{c}(\mathcal{R} / \mathcal{S})}$ can be oriented via $\succeq_{\text {mul }}^{c}$.

Lemma 5.11. For any signature $\mathcal{F}$ and $c \in \mathbb{N}$ it holds that $\rightarrow_{\text {raise }_{c}(\mathcal{F})} \subseteq \succeq_{\text {mul }}^{c}$.

Proof. Assume that there are terms $s$ and $t$ such that $s \rightarrow_{\text {raise }_{c}(\mathcal{F})} t$. According to the definition of $\operatorname{raise}_{c}(\mathcal{F})$ we have $\mathcal{H}(t)=(\mathcal{H}(s) \backslash\{d\}) \cup\{d+1\}$ for some height $d<_{\mathbb{N}} c$. Thus $s \succ_{\text {mul }}^{c} t$ and hence $s \succeq_{\text {mul }}^{c} t$ according to the definition of $\succeq_{\text {mul }}^{c}$.

Using Lemma 5.11 it is easy to extend Theorem 5.7 to TRSs that are non-linear but non-duplicating.

Theorem 5.12. Let $\mathcal{R} / \mathcal{S}$ be a non-duplicating relative TRS and $\mathcal{R}$ be non-collapsing. If $\mathcal{R} / \mathcal{S}$ is match-raise-RT-bounded for a language $L$ then $\mathcal{R} / \mathcal{S}$ is terminating on L. Furthermore, $\mathrm{cp}_{L}\left(n, \rightarrow_{\mathcal{R} / \mathcal{S}}\right)=\mathcal{O}(n)$.

Proof. First we show that $\mathcal{R} / \mathcal{S}$ is terminating on $L$. Assume to the contrary that there is an infinite rewrite sequence of the form

$$
t_{1} \rightarrow_{\mathcal{R} / \mathcal{S}} t_{2} \rightarrow_{\mathcal{R} / \mathcal{S}} t_{3} \rightarrow_{\mathcal{R} / \mathcal{S}} \cdots
$$

with $t_{1} \in L$. Let $\mathcal{R} / \mathcal{S}$ be match-raise-RT-bounded for $L$ by a $c \in \mathbb{N}$. Lemma 5.10 yields an infinite $\stackrel{r}{\rightarrow}_{\text {match- } R T^{c}(\mathcal{R} / \mathcal{S})}$ rewrite sequence

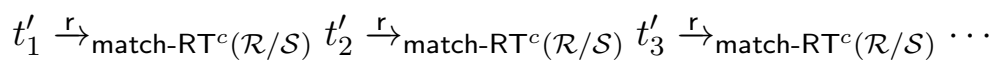

starting from $t_{1}^{\prime}=\operatorname{lift}_{0}\left(t_{1}\right)$ such that base $\left(t_{i}^{\prime}\right)=t_{i}$ for all $i \geqslant 1$. Because $\mathcal{R} / \mathcal{S}$ is match-raiseRT-bounded for $L$ by $c$, the height of every function symbol occurring in a term in the lifted sequence is at most $c$. Hence the employed rewrite rules in the derivation emanating from $t_{1}^{\prime}$ must come from match- $\mathrm{RT}_{c}(\mathcal{R} / \mathcal{S})$. With help of Lemmata 5.6 and 5.11, transitivity of $\succeq_{\text {mul }}^{c}$, and compatibility of $\succ_{\text {mul }}^{c}$ and $\succeq_{\text {mul }}^{c}$ we deduce that $t_{i}^{\prime} \succ_{\text {mul }}^{c} t_{i+1}^{\prime}$ for all $i \geqslant 1$. (Note that Lemma 5.6 requires that $\mathcal{R} / \mathcal{S}$ is non-duplicating.) However, this is excluded because $<_{\mathbb{N}}$ is well-founded on $\{0, \ldots, c\}$ and hence $\succ_{\text {mul }}^{c}$ is well-founded on $\mathcal{T}\left(\mathcal{F}_{\{0, \ldots, c\}}, \mathcal{V}\right)$.

To prove the second part of the theorem, consider an arbitrary (terminating) rewrite sequence

$$
u \rightarrow_{\mathcal{R} / \mathcal{S}} u_{1} \rightarrow_{\mathcal{R} / \mathcal{S}} \cdots \rightarrow_{\mathcal{R} / \mathcal{S}} u_{m}
$$

with $u \in L$. Similar as before this rewrite sequence can be lifted to a $\stackrel{r}{\rightarrow}_{\text {match-RT }}(\mathcal{R} / \mathcal{S})^{-}$ sequence of the same length

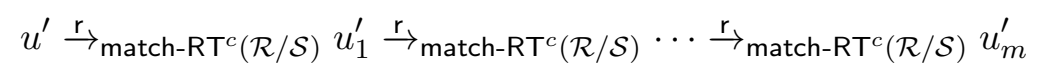

such that $u^{\prime}=\operatorname{lift}_{0}(u)$ and $u_{i}^{\prime} \succ_{\text {mul }}^{c} u_{i+1}^{\prime}$ for all $i \in\{0, \ldots, m-1\}$. Here $u_{0}^{\prime}=u^{\prime}$ and $c \in \mathbb{N}$ such that the relative TRS $\mathcal{R} / \mathcal{S}$ is match-raise-RT-bounded for $L$ by $c$. Similar as in the proof of Theorem 5.1 we can conclude that the length of the $\mathcal{R} / \mathcal{S}$-rewrite sequence starting at the term $u$ is bounded by $\|u\| \cdot(k+1)^{c}$ where $k$ is the maximal number of function symbols occurring in some right-hand side in $\mathcal{R} \cup \mathcal{S}$; just replace $\succ_{\text {mul }}$ by $\succ_{\text {mul }}^{c}$. 
5.4. Automation. To automatically prove that a given relative TRS is match(-raise)-RTbounded for some language $L$ we use (quasi-deterministic, raise-consistent, and) compatible tree automata. Here a tree automaton $\mathcal{A}$ is said to be compatible with a relative TRS $\mathcal{R} / \mathcal{S}$ and a language $L$ if $\mathcal{A}$ is compatible with $\mathcal{R} \cup \mathcal{S}$ and $L$.

Lemma 5.13. Let $\mathcal{R} / \mathcal{S}$ be a left-linear relative $T R S, L$ a language, and $c \in \mathbb{N}$. Let $\mathcal{A}$ be a tree automaton. If $\mathcal{A}$ is compatible with the relative TRS match-RT ${ }^{c}(\mathcal{R} / \mathcal{S})$ and $\operatorname{lift}_{0}(L)$ such that the height of each function symbol occurring in transitions in $\mathcal{A}$ is at most $c$ then $\mathcal{R} / \mathcal{S}$ is match-RT-bounded for $L$.

Proof. Easy consequence of Definition 5.4 and the fact that compatible tree automata are closed under left-linear rewriting.

In case of non-left-linear TRSs we obtain the following result.

Lemma 5.14. Let $\mathcal{R} / \mathcal{S}$ be a relative $T R S, L$ a language, and $c \in \mathbb{N}$. Let $\mathcal{A}$ be a quasideterministic and raise-consistent tree automaton. If $\mathcal{A}$ is compatible with match-RT $\mathrm{T}^{c}(\mathcal{R} / \mathcal{S})$ and $\operatorname{lift}_{0}(L)$ such that the height of each function symbol occurring in transitions in $\mathcal{A}$ is at most $c$ then $\mathcal{R} / \mathcal{S}$ is match-raise-RT-bounded for $L$.

Proof. Straightforward by using the fact that quasi-deterministic, raise-consistent and compatible tree automata are closed under rewriting.

To prove that a relative TRS $\mathcal{R} / \mathcal{S}$ is match(-raise)-RT-bounded for a set of terms $L$ we construct a (quasi-deterministic and raise-consistent) tree automaton $\mathcal{A}=\left(\mathcal{F}, Q, Q_{f}, \Delta\right)$ that is compatible with the rewrite rules of match-RT ${ }^{c}(\mathcal{R} / \mathcal{S})$ and $\operatorname{lift}_{0}(L)$. Since the set $\rightarrow_{\text {match-RT }}^{*}(\mathcal{R} / \mathcal{S})\left(\operatorname{lift}_{0}(L)\right)$ need not be regular, even for left-linear $\mathcal{R}$ and $\mathcal{S}$ and regular $L$ (see [8]) we cannot hope to give an exact automaton construction. The general idea [7,8] is to look for violations of the compatibility requirement: $l \sigma \rightarrow_{\Delta}^{*} q\left(l \sigma \rightarrow_{\Delta_{d}}^{*} q\right)$ and $r \sigma t_{\Delta}^{*} q$ for some rewrite rule $l \rightarrow r$, state substitution $\sigma: \operatorname{Var}(l) \rightarrow Q\left(\sigma: \operatorname{Var}(l) \rightarrow Q_{d}\right)$, and state $q \in Q\left(q \in Q_{d}\right)$. Then we add new states and transitions to the current automaton to ensure $r \sigma \rightarrow_{\Delta}^{*} q$. After $r \sigma \rightarrow_{\Delta}^{*} q$ has been established, we repeat this process until a (quasi-deterministic, raise-consistent, and) compatible automaton is obtained. Note that this may never happen if new states are repeatedly added. To guess an appropriate $c$ we start with $c=0$. As soon as a new transition $f_{d}\left(q_{1}, \ldots, q_{n}\right) \rightarrow q$ with $d>_{\mathbb{N}} c$ is added to the constructed tree automaton, we set $c=d$ and proceed with the construction.

Example 5.15. We show that the relative TRS $\mathcal{R} / \mathcal{S}$ of Example 5.3 over the signature $\mathcal{F}=\left\{\right.$ nil, cons, rev, rev $\left.^{\prime}\right\}$ is match-RT-bounded for $\mathcal{T}(\mathcal{F})$ by constructing a compatible tree automaton. As starting point we consider the initial tree automaton

$$
\text { nil }_{0} \rightarrow 1 \quad \operatorname{cons}_{0}(1,1) \rightarrow 1 \quad \operatorname{rev}_{0}(1) \rightarrow 1 \quad \operatorname{rev}_{0}^{\prime}(1,1) \rightarrow 1
$$

which accepts all ground terms over the enriched signature $\operatorname{lift}_{0}(\mathcal{F})$. The first compatibility violation we consider is caused by the rewrite $\operatorname{rule} \operatorname{rev}_{0}(x) \rightarrow_{\operatorname{match}(\mathcal{R})} \operatorname{rev}_{1}^{\prime}\left(x, \operatorname{nil}_{1}\right)$. We have $\operatorname{rev}_{0}(1) \rightarrow 1$ but not $\operatorname{rev}_{1}^{\prime}\left(1\right.$, nil $\left._{1}\right) \rightarrow^{*} 1$. To solve this violation we add the transitions nil $\rightarrow 2$ and $\operatorname{rev}_{1}^{\prime}(1,2) \rightarrow 1$. The compatibility violation caused by the rewrite rule $\operatorname{rev}_{1}^{\prime}\left(\right.$ nil $\left._{0}, y\right) \rightarrow_{\text {match-RT}}(\mathcal{S}) y$ and the derivation $\operatorname{rev}_{1}^{\prime}\left(\right.$ nil $\left._{0}, 2\right) \rightarrow^{*} 1$ is solved by adding the transition $2 \rightarrow 1$. Note that we are currently using match- $\mathrm{RT}^{1}(\mathcal{S})$ because the maximal height of a function symbol occurring in the underlying tree automaton is 1 . Next we

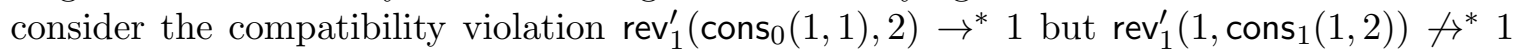


induced by the rule $\operatorname{rev}_{1}^{\prime}\left(\operatorname{cons}_{0}(x, y), z\right) \rightarrow_{\text {match-RT}}{ }^{1}(\mathcal{S}) \operatorname{rev}_{1}^{\prime}\left(y, \operatorname{cons}_{1}(x, z)\right)$. In order to ensure $\operatorname{rev}_{1}^{\prime}\left(1, \operatorname{cons}_{1}(1,2)\right) \rightarrow^{*} 1$ we reuse the transition $\operatorname{rev}_{1}^{\prime}(1,2) \rightarrow 1$ and add the new transition cons $_{1}(1,2) \rightarrow 2$. Finally, $\operatorname{rev}_{0}^{\prime}\left(\operatorname{cons}_{1}(1,2), 1\right) \rightarrow^{*} 1$ and $\operatorname{rev}_{0}^{\prime}\left(\operatorname{cons}_{1}(x, y), z\right) \rightarrow_{\text {match-RT }}(\mathcal{S})$ $\operatorname{rev}_{1}^{\prime}\left(y, \operatorname{cons}_{1}(x, z)\right)$ give rise to the transition $\operatorname{cons}_{1}(1,1) \rightarrow 2$. After this step, the obtained tree automaton is compatible with match- $\mathrm{RT}^{1}(\mathcal{R} / \mathcal{S})$. Hence $\mathcal{R} / \mathcal{S}$ is match-RT-bounded for $\mathcal{T}(\mathcal{F})$ by 1 . Due to Theorem 5.7 we can conclude that $\mathcal{R} / \mathcal{S}$ admits at most linear complexity. We remark that the ordinary match-bound technique (Theorem 5.1) fails on $\mathcal{R} / \mathcal{S}$ because $\mathcal{R} \cup \mathcal{S}$ induces quadratic complexity:

$$
\begin{aligned}
\operatorname{rev}^{n}(x) \sigma^{m} & \rightarrow \operatorname{rev}^{n-1}\left(\operatorname{rev}^{\prime}(x, \operatorname{nil})\right) \sigma^{m} \rightarrow^{m} \operatorname{rev}^{n-1}\left(\operatorname{rev}^{\prime}(\operatorname{nil}, x)\right) \sigma^{m} \\
& \rightarrow \operatorname{rev}^{n-1}(x) \sigma^{m} \rightarrow{ }^{(n-1)(m+2)} x \sigma^{m}
\end{aligned}
$$

with $\sigma=\{x \mapsto \operatorname{cons}(y, x)\})$ for all $n, m \geqslant 1$.

\section{Assessment}

In this section we compare the complexity proving power of the direct and the modular setting on a theoretical level. Gains in power in practice are reported in Section 8 . In the first part of this section we show that for TMIs of dimension one, i.e. SLIs, in theory both approaches are equivalent but in the general case the modular setting allows TMIs of smaller dimensions to succeed. Since the dimension of the TMI corresponds to the degree of the polynomial bound the modular setting allows to establish tighter bounds. The second part of this section shows that the modular setting is strictly more powerful than the direct one, i.e., there are systems where the modular setting admits a complexity proof but all involved methods cannot succeed on its own in the direct setting. To make the presentation easier we assume the original problems to be standard (in contrast to relative) TRSs. This has no effect on the results. The next lemma states that for SLIs in theory there is no difference in power between the two settings.

Lemma 6.1. Let $\mathcal{R}=\mathcal{R}_{1} \cup \mathcal{R}_{2}$ be a TRS. There exists an SLI $\mathcal{M}$ compatible with $\mathcal{R}$ if and only if there exist SLIs $\mathcal{M}_{1}$ and $\mathcal{M}_{2}$ such that $\mathcal{M}_{1}$ is compatible with $\mathcal{R}_{1} / \mathcal{R}_{2}$ and $\mathcal{M}_{2}$ is compatible with $\mathcal{R}_{2} / \mathcal{R}_{1}$.

Proof. The implication from left to right obviously holds since $\mathcal{M}$ is a suitable candidate for $\mathcal{M}_{1}$ and $\mathcal{M}_{2}$. For the reverse direction we construct an SLI $\mathcal{M}$ compatible with $\mathcal{R}$ based on the SLIs $\mathcal{M}_{1}$ and $\mathcal{M}_{2}$. Let $f_{\mathcal{M}_{1}}\left(x_{1}, \ldots, x_{m}\right)=x_{1}+\cdots+x_{m}+f_{1}$ and $f_{\mathcal{M}_{2}}\left(x_{1}, \ldots, x_{m}\right)=$ $x_{1}+\cdots+x_{m}+f_{2}$. It is straightforward to check that $f_{\mathcal{M}}\left(x_{1}, \ldots, x_{m}\right)=x_{1}+\cdots+x_{m}+f_{1}+f_{2}$ for any $f \in \mathcal{F}$ yields an SLI $\mathcal{M}$ compatible with $\mathcal{R}$.

Due to Theorem 3.6 the complexity is not affected when using the modular setting. Hence when using SLIs in theory both approaches can prove the same bounds. But experiments in Section 8 show that in practice proofs are easier to find in the modular setting since, e.g., the coefficients of the interpretations can be chosen smaller (cf. the proof of Lemma 6.1). If TMIs of larger dimensions are applied then just the only-if direction of Lemma 6.1 holds. This is shown with the help of the next example.

Example 6.2. Consider the TRS $\mathcal{R}$ (Strategy_removed_AG01/\#4.21) consisting of the rules:

$$
1: \mathrm{f}(1) \rightarrow \mathrm{f}(\mathrm{g}(1)) \quad 2: \mathrm{f}(\mathrm{f}(x)) \rightarrow \mathrm{f}(x) \quad 3: \mathrm{g}(0) \rightarrow \mathrm{g}(\mathrm{f}(0)) \quad 4: \mathrm{g}(\mathrm{g}(x)) \rightarrow \mathrm{g}(x)
$$


The TMIs $\mathcal{M}_{1}$

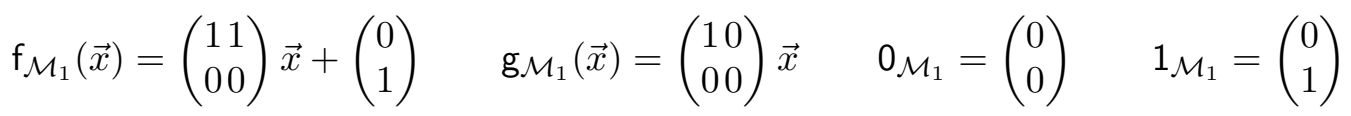

and $\mathcal{M}_{2}$

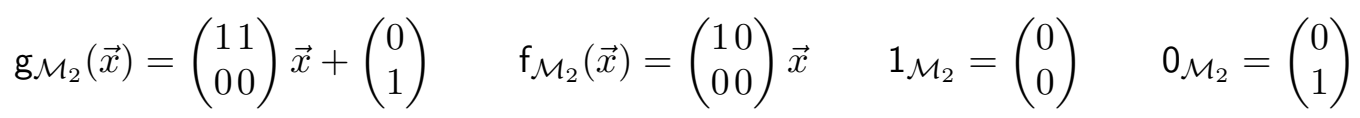

show quadratic upper bounds on the derivational complexity of the systems $\{3,4\} /\{1,2\}$ and $\{1,2\} /\{3,4\}$, respectively. Theorem 3.6 establishes a quadratic upper bound for $\mathcal{R}$.

Although for the TRS in Example6.2 TMIs of dimension two could establish a quadratic upper bound on the derivational complexity in the modular setting, they cannot do so in the direct setting because of the next lemma. (We remark that there exist TMIs of dimension three that are compatible with this TRS).

Lemma 6.3. The TRS Strategy_removed_AG01/\#4.21 does not admit a TMI of dimension two compatible with it.

Proof. To address all possible interpretations we extracted the set of constraints that represent a TMI of dimension two compatible with the TRS Strategy_removed_AG01/\#4.21. MiniSmt [33] can detect unsatisfiability of these constraints. Details of this proof can be found at the web site in Footnote 6 on page 29.

The next result shows that any direct proof transfers into the modular setting without increasing the bounds on the complexity.

Lemma 6.4. Let $\succ$ be a finitely branching rewrite relation and let $\mathcal{R}=\mathcal{R}_{1} \cup \mathcal{R}_{2}$ be a TRS compatible with $\succ$. Then there exist complexity pairs $\left(\succ_{1}, \succeq_{1}\right)$ and $\left(\succ_{2}, \succeq_{2}\right)$ which are compatible with the relative TRSs $\mathcal{R}_{1} / \mathcal{R}_{2}$ and $\mathcal{R}_{2} / \mathcal{R}_{1}$, respectively. Furthermore for any language $L$ we have $\mathrm{cp}_{L}(n, \succ)=\Theta\left(\mathrm{cp}_{L}\left(n, \succ_{1}\right)+\mathrm{cp}_{L}\left(n, \succ_{2}\right)\right)$.

Proof. Fix $i$. Let $\left(\succ_{i}, \succeq_{i}\right)$ be $(\succ,=)$. It is easy to see that $(\succ,=)$ is a complexity pair because $\succ$ and $=$ are compatible rewrite relations. It remains to show that for any term $t \in L$ we have $\mathrm{cp}_{L}(n, \succ)=\Theta\left(\mathrm{cp}_{L}\left(n, \succ_{1}\right)+\mathrm{cp}_{L}\left(n, \succ_{2}\right)\right)$. To this end we observe that $\operatorname{dh}\left(t, \succ_{1}\right)+\operatorname{dh}\left(t, \succ_{2}\right)=2 \cdot \operatorname{dh}(t, \succ)$ for all terms $t \in L$. Basic properties of the $\mathcal{O}$-notation yield the desired result.

Due to Example 6.2 and Lemmata 6.3 and 6.4 we obtain that the modular setting allows to use TMIs of smaller dimensions than the direct one, which allows to establish tighter bounds. The next example (together with Lemma 6.4) shows that in theory the modular complexity setting is strictly more powerful than the direct one since it allows to combine different criteria to establish an upper complexity bound while any method on its own cannot succeed.

Example 6.5. Consider the TRS $\mathcal{R}$ (Transformed_CSR_04/Ex16_Luc06_GM) consisting of the rules:

$$
\begin{aligned}
& 1: \mathrm{c} \rightarrow \mathrm{a} \quad 3: \operatorname{mark}(\mathrm{a}) \rightarrow \mathrm{a} \quad 5: \mathrm{g}(x, y) \rightarrow \mathrm{f}(x, y) \\
& 2: \mathrm{c} \rightarrow \mathrm{b} \quad 4: \operatorname{mark}(\mathrm{b}) \rightarrow \mathrm{c} \quad 6: \mathrm{g}(x, x) \rightarrow \mathrm{g}(\mathrm{a}, \mathrm{b}) \quad 7: \operatorname{mark}(\mathrm{f}(x, y)) \rightarrow \mathrm{g}(\operatorname{mark}(x), y)
\end{aligned}
$$


The following SLI $\mathcal{M}$

$$
\mathrm{a}_{\mathcal{M}}=0 \quad \mathrm{~b}_{\mathcal{M}}=0 \quad \mathrm{c}_{\mathcal{M}}=1 \quad \mathrm{f}_{\mathcal{M}}(x, y)=x+y \quad \mathrm{~g}_{\mathcal{M}}(x, y)=x+y+1 \quad \operatorname{mark}_{\mathcal{M}}(x)=x+2
$$

allows Corollary 4.7 to transform the TRS $\mathcal{R}$ into the relative TRS $\{6,7\} /\{1,2,3,4,5\}$. This problem can be split according to Theorem 3.6 into the two relative TRSs $\{6\} /\{1,2,3,4,5,7\}$ and $\{7\} /\{1,2,3,4,5,6\}$. Match-bounds (Theorem 5.12) can show a linear upper bound on the first problem. The following TMI $\mathcal{M}$

$$
\mathrm{a}_{\mathcal{M}}=\left(\begin{array}{l}
0 \\
0
\end{array}\right) \quad \mathrm{f}_{\mathcal{M}}(\vec{x}, \vec{y})=\vec{x}+\left(\begin{array}{l}
10 \\
0
\end{array}\right) \vec{y}+\left(\begin{array}{l}
0 \\
1
\end{array}\right) \quad \operatorname{mark}_{\mathcal{M}}(\vec{x})=\left(\begin{array}{l}
1 \\
01
\end{array}\right) \vec{x}
$$

where $\mathrm{a}_{\mathcal{M}}=\mathrm{b}_{\mathcal{M}}=\mathrm{c}_{\mathcal{M}}$ and $\mathrm{f}_{\mathcal{M}}(\vec{x}, \vec{y})=\mathrm{g}_{\mathcal{M}}(\vec{x}, \vec{y})$ gives a quadratic upper bound on the second relative TRS, establishing a quadratic upper bound on the derivational complexity of $\mathcal{R}$. The quadratic bound is tight as $\mathcal{R}$ admits derivations

$$
\operatorname{mark}^{n}(x) \sigma^{m} \rightarrow^{m} \operatorname{mark}^{n-1}(x) \tau^{m} \gamma \rightarrow^{m} \operatorname{mark}^{n-1}(x) \sigma^{m} \gamma \rightarrow^{2 m(n-1)} x \sigma^{m} \gamma^{n}
$$

of length $2 m n$ where $\sigma=\{x \mapsto \mathrm{f}(x, y)\}, \tau=\{x \mapsto \mathrm{g}(x, y)\}$, and $\gamma=\{x \mapsto \operatorname{mark}(x)\}$. Last but not least we remark that none of the involved techniques can establish an upper bound on its own. In case of match-bounds this follows from the fact that $\mathcal{R}$ admits quadratic derivational complexity. The same reason also holds for Corollary 4.7 because SLIs induce linear complexity bounds. Finally, TMIs fail because they cannot orient the rewrite rule $\mathrm{g}(x, x) \rightarrow \mathrm{g}(\mathrm{a}, \mathrm{b})$.

Hence we obtain the following corollary.

Corollary 6.6. The modular complexity setting is strictly more powerful than the direct one.

Proof. By Lemma 6.4 and Example 6.5.

Next we consider the TRS Zantema_04/z086. The question about the derivational complexity of it has been stated as problem \#105 on the RTA LooP 4

Example 6.7. Consider the TRS $\mathcal{R}$ (Zantema_04/z086) consisting of the rules:

$$
1: \mathrm{a}(\mathrm{a}(x)) \rightarrow \mathrm{c}(\mathrm{b}(x)) \quad 2: \mathrm{b}(\mathrm{b}(x)) \rightarrow \mathrm{c}(\mathrm{a}(x)) \quad 3: \mathrm{c}(\mathrm{c}(x)) \rightarrow \mathrm{b}(\mathrm{a}(x))
$$

Adian [1] showed that $\mathcal{R}$ admits at most quadratic derivational complexity. Since the proof is based on a low-level reasoning on the structure of $\mathcal{R}$, it is specific to this TRS and challenging for automation. With our approach we cannot prove the quadratic bound on the derivational complexity of $\mathcal{R}$. However, Corollary 4.7 permits to establish some progress. Using an SLI counting a's and b's, it suffices to determine the derivational complexity of $\{3\} /\{1,2\}$. This means that $\mathrm{c}(\mathrm{c}(x)) \rightarrow \mathrm{b}(\mathrm{a}(x))$ relative to the other rules dominates the derivational complexity of $\mathcal{R}$. The benefit is that now, e.g., a TMI must only orient one rule strictly and the other two rules weakly (compared to all three rules strictly). It has to be clarified if the relative formulation of the problem can be used to simplify the proof in [1].

The next example shows that although the modular approach often allows to establish lower bounds compared to the direct one, further criteria for splitting TRSs should be investigated.

\footnotetext{
4 http://rtaloop.mancoosi.univ-paris-diderot.fr
} 
Example 6.8. Consider the TRS $\mathcal{R}$ (SK90/4.30) consisting of the following rules:

$$
\begin{array}{lll}
1: \mathrm{f}(\text { nil }) \rightarrow \text { nil } & 3: \mathrm{f}(\text { nil } \circ y) \rightarrow \text { nil } \circ \mathrm{f}(y) & 5: \mathrm{f}((x \circ y) \circ z) \rightarrow \mathrm{f}(x \circ(y \circ z)) \\
2: \mathrm{g}(\text { nil }) \rightarrow \text { nil } & 4: \mathrm{g}(x \circ \text { nil }) \rightarrow \mathrm{g}(x) \circ \text { nil } & 6: \mathrm{g}(x \circ(y \circ z)) \rightarrow \mathrm{g}((x \circ y) \circ z)
\end{array}
$$

In [20] a TMI compatible with $\mathcal{R}$ of dimension four is given showing that the derivational complexity is bounded by a polynomial of degree four. Using Theorem 3.6 with TMIs of dimension three yields a cubic upper bound, i.e., the TMI $\mathcal{M}_{1}$

$$
\begin{aligned}
& \circ_{\mathcal{M}_{1}}(\vec{x}, \vec{y})=\left(\begin{array}{lll}
1 & 0 & 0 \\
0 & 0 & 1 \\
0 & 0 & 1
\end{array}\right) \vec{x}+\vec{y}+\left(\begin{array}{l}
0 \\
0 \\
1
\end{array}\right)
\end{aligned}
$$

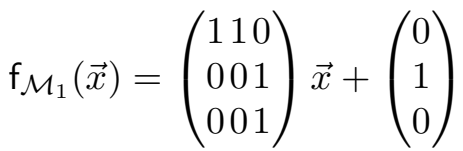

$$
\begin{aligned}
& \mathrm{g}_{\mathcal{M}_{1}}(\vec{x})=\left(\begin{array}{lll}
1 & 0 & 0 \\
0 & 0 & 1 \\
0 & 0 & 1
\end{array}\right) \vec{x} \\
& \text { nil }_{\mathcal{M}_{1}}=\left(\begin{array}{l}
1 \\
1 \\
1
\end{array}\right)
\end{aligned}
$$

yields a cubic upper bound on $\{1,2,3\} /\{4,5,6\}$. So does the TMI $\mathcal{M}_{2}$ with

$$
\mathrm{f}_{\mathcal{M}_{2}}(\vec{x})=\mathrm{g}_{\mathcal{M}_{1}}(\vec{x}) \quad \mathrm{g}_{\mathcal{M}_{2}}(\vec{x})=\mathrm{f}_{\mathcal{M}_{1}}(\vec{x}) \quad \circ_{\mathcal{M}_{2}}(\vec{x}, \vec{y})=\circ_{\mathcal{M}_{1}}(\vec{y}, \vec{x}) \quad \text { nil }_{\mathcal{M}_{2}}=\text { nil }_{\mathcal{M}_{1}}
$$

for $\{4,5,6\} /\{1,2,3\}$. Our approach enables showing a lower complexity than [20] but the derivational complexity of $\mathcal{R}$ is quadratic (see [20]). The quadratic lower bound is justified as $\mathcal{R}$ admits derivations

$$
\mathrm{f}^{n}(x) \sigma^{m} \rightarrow^{n} \text { nil } \circ \mathrm{f}^{n}(x) \sigma^{m-1} \rightarrow^{n} \cdots \rightarrow^{n} x \sigma^{m} \tau^{n}
$$

of length $n m$ where $\sigma=\{x \mapsto$ nil $\circ x\}$ and $\tau=\{x \mapsto \mathrm{f}(x)\}$. We stress that the recent approach in [28] allows to establish a quadratic upper bound. For a comment on the integration of this method into our setting we refer to Section 9 ,

\section{IMPLEMENTATION}

In Section 7.1 we first show how the various theorems from the previous sections can be implemented to obtain some complexity proof. Afterwards Section 7.2 is concerned with lowering the bounds starting from an existing complexity proof.

7.1. Establishing Bounds. To estimate the complexity of a TRS $\mathcal{R}$ with respect to a language $L$, we first transform $\mathcal{R}$ into the relative TRS $\mathcal{R} / \varnothing$. Obviously $\mathrm{cp}_{L}\left(n, \rightarrow_{\mathcal{R}}\right)=$

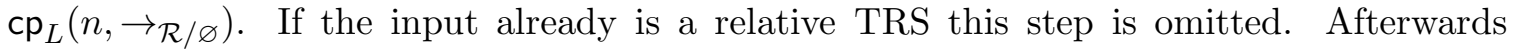
for a relative TRS $\mathcal{R} / \mathcal{S}$ we try to establish a bound on the complexity of $\mathcal{R}_{2} /\left(\mathcal{R}_{1} \cup \mathcal{S}\right)$ with respect to $L$ for some $\mathcal{R}_{1}, \mathcal{R}_{2}$ with $\mathcal{R}_{1}=\mathcal{R} \backslash \mathcal{R}_{2}$ and continue with the relative TRS $\mathcal{R}_{1} /\left(\mathcal{R}_{2} \cup \mathcal{S}\right)$. This step is executed repeatedly until the remaining problem equals $\varnothing /(\mathcal{R} \cup \mathcal{S})$. Then the complexity of $\mathcal{R} / \mathcal{S}$ with respect to $L$ is obtained by summing up all intermediate bounds. In order to establish a maximal number of complexity proofs we run all techniques from Sections 4 and 5 in parallel and the first technique that can shift some rules is used to achieve progress.

Note that the procedure sketched above contains an implicit application of Theorem 3.6. i.e., some method immediately proves a bound for $\mathcal{R}_{2} /\left(\mathcal{R}_{1} \cup \mathcal{S}\right)$ and leaves $\mathcal{R}_{1} /\left(\mathcal{R}_{2} \cup \mathcal{S}\right)$ as open proof obligation. In contrast to an explicit application of Theorem 3.6, here the method that establishes the bound on $\mathcal{R}_{2} /\left(\mathcal{R}_{1} \cup \mathcal{S}\right)$ can select the decomposition of $\mathcal{R}$ into $\mathcal{R}_{1}$ and $\mathcal{R}_{2}$ which is beneficial for performance. As an immediate consequence, proof trees 
degenerate to lists (cf. Example 7.8). In the following we describe the presented approach more formal and refer to it as the complexity framework.

Definition 7.1. A complexity problem (CP problem for short) is a pair $(\mathcal{R} / \mathcal{S}, L)$ consisting of a relative TRS $\mathcal{R} / \mathcal{S}$ and a language $L$.

To operate on CP problems so called complexity processors are used. Similar as in the dependency pair framework we distinguish between sound and complete processors. Here sound complexity processors are used to prove an upper bound on the complexity of a given $\mathrm{CP}$ problem whereas complete complexity processors are applied to derive lower bounds on the complexity.

Definition 7.2. A complexity processor (CP processor for short) is a function that takes a CP problem $(\mathcal{R} / \mathcal{S}, L)$ as input and returns a set of pairs $\bigcup_{1 \leqslant i \leqslant m}\left\{\left(\left(\mathcal{R}_{i} / \mathcal{S}_{i}, L_{i}\right), f_{i}\right)\right\}$ as output 5 Here $\left(\mathcal{R}_{i} / \mathcal{S}_{i}, L_{i}\right)$ is a complexity problem and $f_{i}: \mathbb{N} \rightarrow \mathbb{N}$ for each $1 \leqslant i \leqslant m$. A complexity processor is sound if

$$
\mathrm{cp}_{L}\left(n, \rightarrow_{\mathcal{R} / \mathcal{S}}\right)=\mathcal{O}\left(f_{1}(n)+\cdots+f_{m}(n)+\mathrm{cp}_{L_{1}}\left(n, \rightarrow_{\mathcal{R}_{1} / \mathcal{S}_{1}}\right)+\cdots+\mathrm{cp}_{L_{m}}\left(n, \rightarrow_{\mathcal{R}_{m} / \mathcal{S}_{m}}\right)\right)
$$

and it is called complete if

$$
\mathrm{cp}_{L}\left(n, \rightarrow_{\mathcal{R} / \mathcal{S}}\right)=\Omega\left(f_{1}(n)+\cdots+f_{m}(n)+\mathrm{cp}_{L_{1}}\left(n, \rightarrow_{\mathcal{R}_{1} / \mathcal{S}_{1}}\right)+\cdots+\mathrm{cp}_{L_{m}}\left(n, \rightarrow_{\mathcal{R}_{m} / \mathcal{S}_{m}}\right)\right)
$$

holds.

In the sequel zero denotes the constant zero function, i.e., zero: $\mathbb{N} \rightarrow \mathbb{N}$ with zero $(n)=0$. Next we list some CP processors that can be derived from the previous sections. The first one is based on complexity pairs and can, e.g., be implemented by Theorems 4.1 and 4.3 ,

Theorem 7.3. The CP processor

$$
(\mathcal{R} / \mathcal{S}, L) \mapsto \begin{cases}\left\{\left(\mathcal{R}_{1} /\left(\mathcal{R}_{2} \cup \mathcal{S}\right), L, f\right)\right\} & \text { if } \mathcal{R}_{2} /\left(\mathcal{R}_{1} \cup \mathcal{S}\right) \text { is compatible } \\ \{(\mathcal{R} / \mathcal{S}, L, \text { zero })\} & \text { with a complexity pair }(\succ, \succeq)\end{cases}
$$

where $\mathcal{R}=\mathcal{R}_{1} \cup \mathcal{R}_{2}$, and $f(n)=\operatorname{cp}_{L}(n, \succ)$ is sound.

Proof. Follows from Corollary 3.3 and Theorem 3.6 .

The above processor is implemented by demanding that all rules in $\mathcal{R} \cup \mathcal{S}$ are weakly oriented while at least one rule in $\mathcal{R}$ is oriented strictly. Hence the decomposition of $\mathcal{R}$ into $\mathcal{R}_{1}$ and $\mathcal{R}_{2}$ is performed automatically. The next CP processor requires a mild condition on $\mathcal{R}_{1}$ only. Again the decomposition of $\mathcal{R}$ into $\mathcal{R}_{1}$ and $\mathcal{R}_{2}$ is performed automatically since in the implementation we just demand that the $\mathcal{S}$-rules are weakly oriented while the $\mathcal{R}$-rules may increase by a constant factor and at least one of the rules in $\mathcal{R}$ is oriented strictly.

Theorem 7.4. The CP processor

$$
(\mathcal{R} / \mathcal{S}, L) \mapsto \begin{cases}\left\{\left(\mathcal{R}_{1} /\left(\mathcal{R}_{2} \cup \mathcal{S}\right), L, f\right)\right\} & \text { if } \mathcal{M} \text { is a matrix interpretation with constant } \\ \{(\mathcal{R} / \mathcal{S}, L, \text { zero })\} & \text { growth, } \mathcal{R}_{1} \subseteq \succeq_{\mathcal{M}}^{\text {ncp }} \text { and } \mathcal{R}_{2} / \mathcal{S} \text { is compatible with } \mathcal{M} \\ \text { otherwise }\end{cases}
$$

where $\mathcal{R}=\mathcal{R}_{1} \cup \mathcal{R}_{2}$, and $f(n)=n$ is sound.

Proof. Follows from Theorem 3.6 and Theorem 4.11 .

\footnotetext{
${ }^{5}$ For reasons of readability we write pairs $\left(\left(\mathcal{R}_{i} / \mathcal{S}_{i}, L_{i}\right), f_{i}\right)$ as triples $\left(\mathcal{R}_{i} / \mathcal{S}_{i}, L_{i}, f_{i}\right)$.
} 
The next CP processor is based on match-bounds.

Theorem 7.5. The CP processor

$$
(\mathcal{R} / \mathcal{S}) \mapsto \begin{cases}\left\{\left(\mathcal{R}_{1} /\left(\mathcal{R}_{2} \cup \mathcal{S}\right), L, f\right)\right\} & \text { if } \mathcal{R}_{2} /\left(\mathcal{R}_{1} \cup \mathcal{S}\right) \text { is } \\ & \text { linear and match-RT-bounded for } L \text { or } \\ & \text { non-duplicating and match-raise-RT-bounded for } L \\ \{(\mathcal{R} / \mathcal{S}, L, \text { zero })\} & \text { otherwise }\end{cases}
$$

where $\mathcal{R}=\mathcal{R}_{1} \cup \mathcal{R}_{2}, \mathcal{R}_{2}$ is non-collapsing, and $f(n)=n$ is sound.

Proof. Follows from Theorems 3.6, 5.7, and 5.12,

The above processor is implemented by considering for any rule $l \rightarrow r \in \mathcal{R}$ the decompositions $\mathcal{R}_{2}=\{l \rightarrow r\}$ and $\mathcal{R}_{1}=\mathcal{R} \backslash \mathcal{R}_{2}$ in parallel. The next CP processor we present is not implemented for finding a bound (cf. the discussion at the beginning of the section) but very suitable to tighten existing bounds (see Section 7.2).

Theorem 7.6. The CP processor

$$
(\mathcal{R} / \mathcal{S}, L) \mapsto\left\{\left(\mathcal{R}_{1} /\left(\mathcal{R}_{2} \cup \mathcal{S}\right), L, \text { zero }\right),\left(\mathcal{R}_{2} /\left(\mathcal{R}_{1} \cup \mathcal{S}\right), L, \text { zero }\right)\right\}
$$

where $\mathcal{R}=\mathcal{R}_{1} \cup \mathcal{R}_{2}$ is sound and complete.

Proof. By Theorem 3.6 .

Finally, the main theorem states that the CP framework may be applied to complexity analysis. We say that $P$ is a complexity proof for a relative TRS $\mathcal{R} / \mathcal{S}$ and a language $L$ if all leaves in $P$ are of the shape $\varnothing /(\mathcal{R} \cup \mathcal{S})$.

Theorem 7.7. Let $\mathcal{R} / \mathcal{S}$ be a relative $T R S$ and $L$ be a language. Let $P$ be a complexity proof for $\mathcal{R} / \mathcal{S}$ and $L$ and $f_{1}, \ldots, f_{m}$ be the complexities occurring in this proof. If all $C P$ processors in $P$ are sound then $\mathrm{cp}_{L}\left(n, \rightarrow_{\mathcal{R} / \mathcal{S}}\right)=\mathcal{O}\left(f_{1}(n)+\cdots+f_{m}(n)\right)$. Similarly, if all CP processors in $P$ are complete then $\mathrm{cp}_{L}\left(n, \rightarrow_{\mathcal{R} / \mathcal{S}}\right)=\Omega\left(f_{1}(n)+\cdots+f_{m}(n)\right)$.

Proof. By Definition 7.2 as well as basic properties of $\mathcal{O}$-notation.

We conclude the section with an (abstract) example which illustrates the behavior of the complexity framework.

Example 7.8. Consider the TRS $\mathcal{R}$ of Example 3.7 on page 6 with the complexity proof depicted in Figure 2. After transforming $\mathcal{R}$ into the relative TRS $\mathcal{R} / \varnothing$ the $\mathrm{CP}$ processor of Theorem 7.3 is applied twice. First the (derivational) complexity of the relative TRS $\{1,3,5\} /\{2,4\}$ is estimated by a polynomial of degree five. As a consequence, the rules 1 , 3 , and 5 are moved into the relative component yielding a $\mathrm{CP}$ problem consisting of the relative $\operatorname{TRS}\{2,4\} /\{1,3,5\}$. After that the (derivational) complexity of $\{2,4\} /\{1,3,5\}$ is estimated by a quadratic bound. Since the remaining CP problem is of the shape $\varnothing / \mathcal{R}$ according to Theorem 7.7 the (derivational) complexity of $\mathcal{R}$ is at most quintic. 


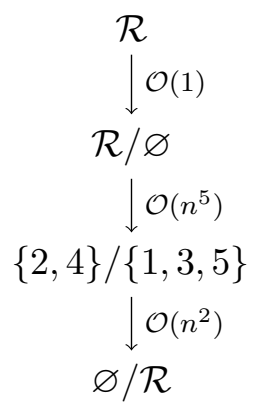

FiguRE 2. Linear complexity proof

7.2. Tightening Bounds. In contrast to termination, which is a plain YES/NO question, complexity corresponds to an optimization problem. Hence automated tools should try to establish as tight bounds as possible. In the direct setting all complexity methods can be executed in parallel and after a fixed amount of time the tightest bound is reported. The next example shows such a case.

Example 7.9. Consider the TRS $\mathcal{R}_{\text {bits }}$ (nontermin/AG01/\#4.28) consisting of the following five rules:

$$
\begin{aligned}
& \text { 1: } \quad \text { half }(0) \rightarrow 0 \\
& \text { 4: } \quad \operatorname{bits}(0) \rightarrow 0 \\
& \text { 2: } \quad \text { half }(\mathrm{s}(0)) \rightarrow 0 \\
& 5: \operatorname{bits}(\mathrm{s}(x)) \rightarrow \mathrm{s}(\operatorname{bits}(\text { half }(\mathrm{s}(x)))) \\
& 3: \text { half }(\mathrm{s}(\mathrm{s}(x))) \rightarrow \mathrm{s}(\text { half }(x))
\end{aligned}
$$

For this TRS the complexity analyzer (aT (cf. Section 8) finds a proof by root-labeling followed by a TMI of dimension two, establishing a quadratic upper bound within five seconds. However, after 90 seconds the tool finds the following AMI $\mathcal{A}$ that shows a linear upper bound:

$$
\operatorname{bits}_{\mathcal{A}}(\vec{x})=\left(\begin{array}{c}
012 \\
045 \\
067
\end{array}\right) \vec{x} \quad \text { half }_{\mathcal{A}}(\vec{x})=\left(\begin{array}{c}
1-\infty-\infty \\
1-\infty-\infty \\
1-\infty-\infty
\end{array}\right) \vec{x} \quad \mathrm{~s}_{\mathcal{A}}(\vec{x})=\left(\begin{array}{cc}
11-\infty \\
70 & 2 \\
16 & 0
\end{array}\right) \vec{x} \quad 0_{\mathcal{A}}=\left(\begin{array}{c}
0 \\
0 \\
-\infty
\end{array}\right)
$$

So, whenever the tool is allowed more than 90 seconds the linear bound can be reported and if the user sets the global timeout to less, then still the quadratic bound can be output.

In the modular setting this simple idea does not work because two problems emerge. The first problem is that the tool does not know how much time it may spend in a single proof step. If it spends too much then it may not finish the proof within the global time limit and if it spends too little then it can miss a low bound. The second problem is that in the modular setting separate criteria may make statements about the complexity of different rules. The question is then to identify the better bound. The next example demonstrates this scenario.

Example 7.10. Consider the TRSs

$$
1: \mathrm{a}(\mathrm{b}(x)) \rightarrow \mathrm{b}(\mathrm{a}(x)) \quad 2: \mathrm{g}(x, x) \rightarrow \mathrm{g}(\mathrm{c}, \mathrm{d})
$$




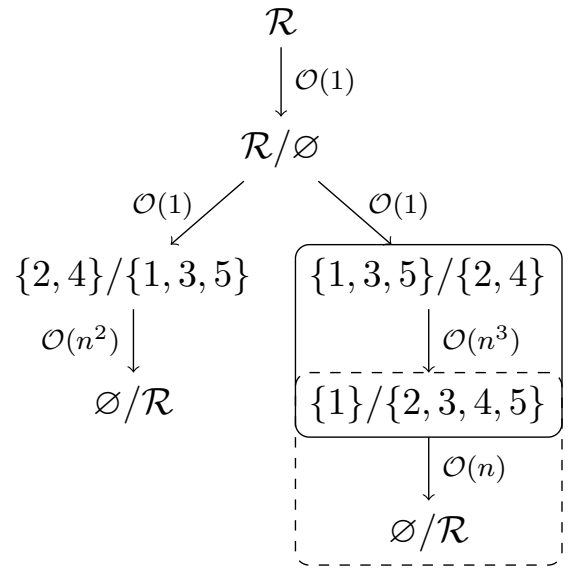

(A) Initial proof

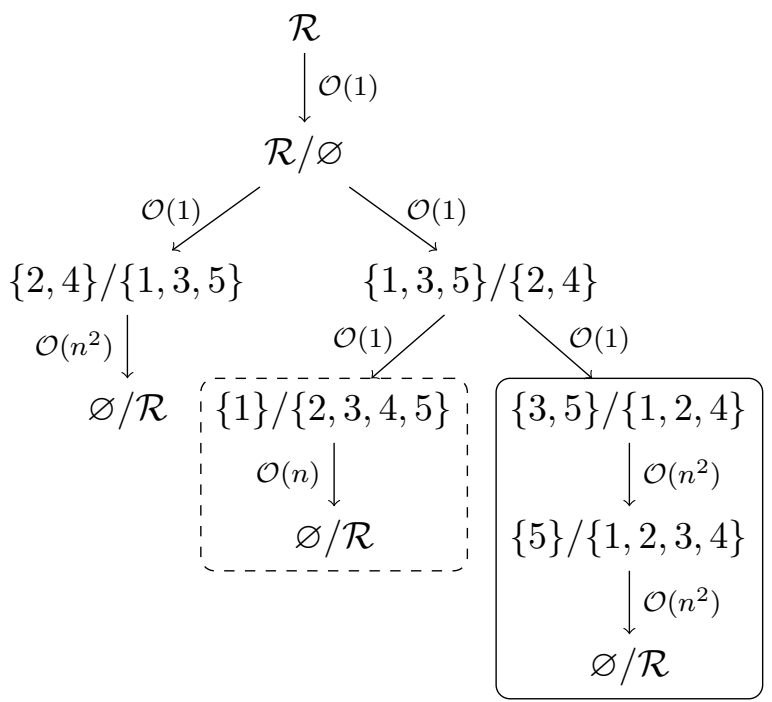

(B) Optimized proof

FiguRE 3. Tightening bounds

The TMI $\mathcal{M}$ with $\operatorname{g}_{\mathcal{M}}(\vec{x}, \vec{y})=\vec{x}+\vec{y}$ and

$$
\mathrm{a}_{\mathcal{M}}(\vec{x})=\left(\begin{array}{l}
11 \\
01
\end{array}\right) \vec{x} \quad \mathrm{~b}_{\mathcal{M}}(\vec{x})=\left(\begin{array}{l}
10 \\
01
\end{array}\right) \vec{x}+\left(\begin{array}{l}
0 \\
1
\end{array}\right) \quad \mathrm{c}_{\mathcal{M}}(\vec{x})=\left(\begin{array}{l}
0 \\
0
\end{array}\right) \quad \mathrm{d}_{\mathcal{M}}(\vec{x})=\left(\begin{array}{l}
0 \\
0
\end{array}\right)
$$

establishes a quadratic upper bound on the complexity of $\{1\} /\{2\}$ whereas match-bounds yield a linear upper bound for $\{2\} /\{1\}$. The question now is with which remaining proof obligation $(\{2\} /\{1\}$ or $\{1\} /\{2\})$ the tool should continue. Note that both bounds are tight.

The following idea overcomes both problems: First we establish some complexity proof according to the procedure described at the beginning of Section 7.1 to obtain a bound for as many systems as possible. Afterwards we optimize this bound. The next example shows how the latter works.

Example 7.11. Consider the TRS $\mathcal{R}$ of Example 3.7 with the complexity proof depicted in Figure 3a, In this exemplary case one part in this proof, highlighted by a solid box, is overestimated by a cubic upper bound. Hence the complexity of the whole system is at most cubic. We remark that this proof step estimates the complexity of $\{3,5\} /\{1,2,4\}$. Now assume that the cubic bound is not optimal, i.e., there exists a proof (that may be longer and harder to find) that induces a quadratic upper bound on the complexity of $\{3,5\} /\{1,2,4\}$. Then the proof is optimized as illustrated in Figure 3b, i.e., $\{1,3,5\} /\{2,4\}$ is split into the problems $\{1\} /\{2,3,4,5\}$ and $\{3,5\} /\{1,2,4\}$ by an application of Theorem 3.6. After that, the proof part of $\{1\} /\{2,3,4,5\}$ is reused in the optimized proof (cf. the dashed boxes in Figure [3a and Figure [3b) whereas the original proof of $\{3,5\} /\{1,2,4\}$ is replaced by the new one, as indicated by the solid box in Figure 3b. Now, the proof in Figure 3b establishes a quadratic upper bound on the complexity of $\mathcal{R}$. For completeness we state that the proof in Figure 3a can be obtained from the linear proof tree shown in Figure 2 by optimization. To show the procedure on a non-linear proof tree this presentation was chosen. 
As the previous example demonstrates the basic idea is to replace single proof steps by new proofs that induce tighter bounds. This procedure is repeated until either the global time limit is reached or none of the bounds can be tightened further. Note that the transformation is sound by Theorem 7.7

The final example in this section shows that it may be easier to find proofs in the modular setting.

Example 7.12. Recall the TRS from Example 7.9. Corollary 4.7 with an SLI that just counts function symbols allows to transform the initial problem into $\{5\} /\{1,2,3,4\}$. The AMI of dimension three with

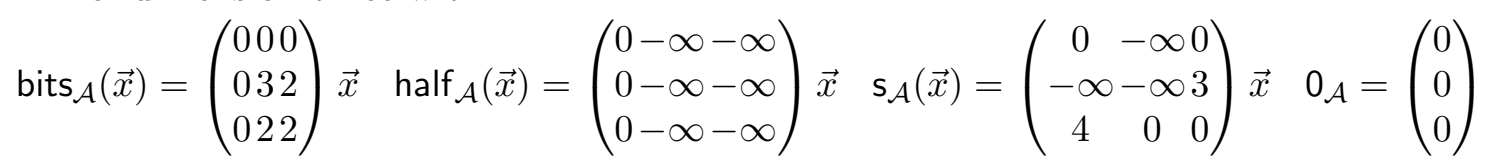

allows to show linear derivational complexity of $\mathcal{R}_{\text {bits. }}$. Note that GT finds this interpretation within three seconds whereas it took the tool 90 seconds to find a suitable interpretation for the direct setting.

\section{Experimental Results}

The techniques described in the preceding sections are implemented in the complexity analyzer GT (freely available from http://cl-informatik.uibk.ac.at/software/cat) which is built on top of $\mathrm{T}_{\mathrm{T}} \mathrm{T}_{2}$ [19], a powerful termination tool for TRSs.

Below we report on the experiment 6 we performed. We considered the 2132 TRSs in version 7.0.2 of the TPDB without strategy or theory annotation. The 1172 non-duplicating systems of this collection have been used for experiments with derivational complexity (note that a duplicating system gives rise to at least exponentially long derivations). For runtime complexity we considered the 1339 systems that are not trivial, e.g., where the set of constructor-based terms is not finite and terminating. In this collection there are 910 non-duplicating systems. All tests have been performed on a server equipped with eight dual-core AMD Opteron ${ }^{\circledR}$ processors 885 running at a clock rate of $2.6 \mathrm{GHz}$ and $64 \mathrm{~GB}$ of main memory. We remark that similar results have been obtained on a dual-core laptop. If a tool did not report an answer within 60 seconds, its execution was aborted.

As complexity preserving transformations we employ uncurrying [29] for applicative systems whenever it applies and root-labeling [23] in parallel to the base methods. As base methods we use the match-bounds technique as well as TMIs [20,21] and AMIs [16] of dimensions one to five. The latter two are implemented by bit-blasting arithmetic operations to SAT [6]. All base methods are run in parallel and started upon program execution.

Our results are summarized in Tables 1 and 2, Here, direct refers to the conventional setting where all rules must be oriented at once whereas modular first transforms a TRS $\mathcal{R}$ into a relative TRS $\mathcal{R} / \varnothing$ before the CP processors from Section 7.1 (except Theorem 7.6) are employed. In the tables the postfix $\star$ indicates that after establishing a bound it is tried to be tightened as explained in Section 7.2. The columns $\mathcal{O}(n), \mathcal{O}\left(n^{2}\right), \ldots, \mathcal{O}\left(n^{k}\right)$ give the number of linear, quadratic, ..., polynomial upper bounds that could be established. We also list the average time (in seconds) needed for finding a bound in the last column. For reference we also give the data for the winners of the corresponding categories in the

\footnotetext{
${ }^{6}$ Full details available from http://cl-informatik.uibk.ac.at/software/cat/10lmcs.
} 
TABLE 1. Derivational complexity of 1172 TRSs

\begin{tabular}{|c|c|c|c|c|c|}
\hline & $\mathcal{O}\left(n^{k}\right)$ & $\mathcal{O}(n)$ & $\mathcal{O}\left(n^{2}\right)$ & $\mathcal{O}\left(n^{3}\right)$ & time \\
\hline direct & 315 & 202 & 234 & 259 & 1.7 \\
\hline direct $\star$ & 315 & 215 & 303 & 312 & 7.9 \\
\hline modular & 334 & 208 & 228 & 261 & 2.6 \\
\hline modularᄎ & 334 & 221 & 321 & 329 & 10.6 \\
\hline (aT & 328 & 216 & 310 & 319 & 4.2 \\
\hline CaT $\star$ & 328 & 219 & 317 & 324 & 11.5 \\
\hline
\end{tabular}

TABLE 2. Runtime complexity of 910 TRSs

\begin{tabular}{|c|c|c|c|c|c|}
\hline & $\mathcal{O}\left(n^{k}\right)$ & $\overline{\mathcal{O}(n)}$ & $\mathcal{O}\left(n^{2}\right)$ & $\mathcal{O}\left(n^{3}\right)$ & time \\
\hline direct & 372 & 354 & 358 & 365 & 0.6 \\
\hline direct $\star$ & 372 & 355 & 370 & 371 & 1.1 \\
\hline modular & 376 & 358 & 359 & 364 & 0.5 \\
\hline modular $\star$ & 376 & 362 & 374 & 375 & 1.2 \\
\hline$\overline{\mathrm{T}_{\mathrm{C}}^{\top}}$ & 354 & 351 & 353 & 354 & 4.8 \\
\hline $\mathrm{T}_{\mathrm{C}}^{\mathrm{T}}(1339)$ & 363 & 360 & 362 & 363 & 4.8 \\
\hline
\end{tabular}

2010 edition of the termination competition. For derivational complexity this is CaT and for runtime complexity this is $\mathrm{T}_{\mathrm{C}}^{\mathrm{T}}[3$.

Table 1 shows the results for derivational complexity. Here the modular approach allows to prove significantly more polynomial bounds (column $\mathcal{O}\left(n^{k}\right)$ ) and furthermore these bounds are also smaller than for the direct approach (especially if tightening of bounds is used). The modular setting is slower since there typically more proofs are required to succeed. The rows postfixed $\star$ prove that refining bounds is beneficial, especially if all criteria are run in parallel, which is essential to maximize the total number of upper bounds. The 2010 version of GT did not use tightening of bounds. To maximize the number of low bounds the tool executes criteria that yield larger complexity bounds slightly delayed. This explains why for CT tightening bounds increases the global performance less compared to direct and modular. On the contrary, GT misses some proofs compared to modular since (costly) criteria are not executed for up to 60 seconds.

Table 2 shows the results for runtime complexity on the 910 TRSs that are nonduplicating and non-trivial. Here, the starting language for the match-bounds technique has been restricted to constructor-based terms, i.e., no defined symbols are allowed below the root. This makes match-bounds a very powerful technique for runtime complexity, explaining the high number of linear bounds 7 We remark that in contrast to the criteria we employ $\mathrm{T}_{\mathrm{C}}^{\mathrm{T}}$ can also estimate polynomial bounds for duplicating systems based on weak dependency pairs [11,12. The row ${ }^{\top} C^{\top}(1339)$ corresponds to ${ }^{\top} C^{\top}$ run on all 1339 TRSs in the benchmark for runtime complexity. Hence this row includes duplicating TRSs. For 9 of these $\mathrm{T}_{\mathrm{C}}^{\mathrm{T}}$ can prove a polynomial upper bound.

\footnotetext{
7 In the 2010 competition GT proved upper bounds on the derivational complexity also in the category for runtime complexity. This explains why our methods here outperform ${ }^{\top}{ }^{\top}$ while in the competition caT came second in this division.
} 
For further comparison with other tools we refer the reader to the international termination competition (referenced in Footnote 1 on page 1). Since 2008, when the complexity categories have been installed in the termination competition, CaT won the division for derivational complexity every year.

\section{Conclusion}

In this article we have introduced a modular approach for estimating the complexity of TRSs by considering relative rewriting. We showed how existing criteria (for full rewriting) can be lifted into the relative setting. The modular approach is easy to implement and has been proved strictly more powerful than traditional methods in theory and practice. Since the modular method allows to combine different criteria, typically smaller complexity bounds are achieved than with the direct setting. Furthermore the modular treatment allows to establish bounds for systems where each of the involved basic methods alone fails. Although originally developed for derivational complexity our results directly apply to more restrictive notions of complexity, e.g., runtime complexity (see also below). Finally we remark that our setting allows a more fine-grained complexity analysis, i.e., while traditionally quadratic derivational complexity ensures that any rule is applied at most quadratically often, our approach can make different statements about single rules. Hence even if a proof attempt does not succeed completely, it may highlight the problematic rules.

We remark that complexity proofs using TMIs (for relative rewriting) can be certified with CeTA [26].

As related work we mention [14] which also considers relative rewriting for complexity analysis. However, there the complexity of $\mathcal{R}_{1} \cup \mathcal{R}_{2}$ is investigated by considering $\mathcal{R}_{1} / \mathcal{R}_{2}$ and $\mathcal{R}_{2}$. Hence [14] also gives rise to a modular reasoning but the obtained complexities are typically beyond polynomials. For runtime complexity analysis Hirokawa and Moser [11,12] consider weak dependency pair steps relative to the usable rules, i.e., $\operatorname{WDP}(\mathcal{R}) / \operatorname{UR}(\mathcal{R})$. However, since in the current formulation of weak dependency pairs some complexity might be hidden in the usable rules they do not really obtain a relative problem. As a consequence they can only apply restricted criteria for the usable rules. Note that our approach can directly be used to show bounds on $\operatorname{WDP}(\mathcal{R}) / \operatorname{UR}(\mathcal{R})$ by considering $\operatorname{WDP}(\mathcal{R}) \cup \operatorname{UR}(\mathcal{R})$. Due to Corollary 4.7 this problem can be transformed into an (unrestricted) relative problem $\operatorname{WDP}(\mathcal{R}) / \operatorname{UR}(\mathcal{R})$ whenever the constraints in [1] are satisfied. Moreover, if somehow the problematic usable rules could be determined and shifted into the $\operatorname{WDP}(\mathcal{R})$ component, then this improved version of weak dependency pairs corresponds to a relative problem without additional restrictions, admitting further benefit from our contributions.

Recently two approaches were proposed which admit polynomially bounded matrix interpretations going beyond TMIs. While [28] considers weighted automata, in [21] (joint) spectral radius theory is employed. For ease of presentation these criteria have not been considered in this work but since both are based on matrix interpretations, they perfectly suit our modular setting.

For future work we plan to investigate criteria that allow to analyze the complexity of a TRS $\mathcal{R}$ by the complexities of $\mathcal{R}_{1}$ and $\mathcal{R}_{2}$ where $\mathcal{R}=\mathcal{R}_{1} \cup \mathcal{R}_{2}$. We anticipate that results from modularity [22] are helpful for this aim. 
Acknowledgments. We thank Johannes Waldmann for directing our attention to Example 4.5 and Martin Avanzini for providing a binary of the 2010 competition version of $\mathrm{T}_{\mathrm{C}} \mathrm{T}$.

\section{REFERENCES}

[1] Adian, S.I.: Upper bound on the derivational complexity in some word rewriting system. Doklady Math. 80(2), 679-683 (2009)

[2] Arts, T., Giesl, J.: Termination of term rewriting using dependency pairs. TCS 236(1-2), 133-178 (2000)

[3] Avanzini, M., Moser, G., Schnabl, A.: Automated implicit computational complexity analysis (system description). In: IJCAR 4. LNCS (LNAI), vol. 5195, pp. 132-138 (2008)

[4] Baader, F., Nipkow, T.: Term Rewriting and All That. Cambridge University Press, Cambridge (1998)

[5] Dershowitz, N., Manna, Z.: Proving termination with multiset orderings. Comm. ACM 22(8), 465-476 (1979)

[6] Endrullis, J., Waldmann, J., Zantema, H.: Matrix interpretations for proving termination of term rewriting. JAR 40(2-3), 195-220 (2008)

[7] Genet, T.: Decidable approximations of sets of descendants and sets of normal forms. In: RTA 1998. LNCS, vol. 1379, pp. 151-165 (1998)

[8] Geser, A., Hofbauer, D., Waldmann, J., Zantema, H.: On tree automata that certify termination of left-linear term rewriting systems. I\&C 205(4), 512-534 (2007)

[9] Geser, A.: Relative termination. PhD thesis, Universität Passau, Germany (1990). Available as: Report 91-03, Ulmer Informatik-Berichte, Universität Ulm, 1991

[10] Hirokawa, N., Middeldorp, A.: Automating the dependency pair method. I\&C 199(1-2), 172-199 (2005)

[11] Hirokawa, N., Moser, G.: Automated complexity analysis based on the dependency pair method. In: IJCAR 4. LNCS, vol. 5195, pp. 364-379 (2008)

[12] Hirokawa, N., Moser, G.: Complexity, graphs, and the dependency pair method. In: LPAR 2008. LNCS (LNAI), vol. 5330, pp. 652-666 (2008)

[13] Hofbauer, D., Lautemann, C.: Termination proofs and the length of derivations (preliminary version). In: RTA 1989. LNCS, vol. 355, pp. 167-177 (1989)

[14] Hofbauer, D., Waldmann, J.: Complexity bounds from relative termination proofs. Talk at the Workshop on Proof Theory and Rewriting, Obergurgl (2006). Available from http://www.imn.htwk-leipzig.de/ waldmann/talk/06/rpt/rel/main.pdf

[15] Koprowski, A., Waldmann, J.: Arctic termination ... below zero. In: RTA 2008. LNCS, vol. 5117, pp. 202-216 (2008)

[16] Koprowski, A., Waldmann, J.: Max/plus tree automata for termination of term rewriting. AC 19(2), 357-392 (2009)

[17] Korp, M., Middeldorp, A.: Proving termination of rewrite systems using bounds. In: RTA 2007. LNCS, vol. 4533, pp. 273-287 (2007)

[18] Korp, M., Middeldorp, A.: Match-bounds revisited. I\&C 207(11), 1259-1283 (2009)

[19] Korp, M., Sternagel, C., Zankl, H., Middeldorp, A.: Tyrolean Termination Tool 2. In: RTA 2009. LNCS, vol. 5595, pp. 295-304 (2009)

[20] Moser, G., Schnabl, A., Waldmann, J.: Complexity analysis of term rewriting based on matrix and context dependent interpretations. In: FSTTCS 2008. LIPIcs, vol. 2, pp. 304-315 (2008)

[21] Neurauter, F., Zankl, H., Middeldorp, A.: Revisiting matrix interpretations for polynomial derivational complexity of term rewriting. In: LPAR 17. LNCS (ARCoSS), vol. 6397, pp. 550-564 (2010)

[22] Ohlebusch, E.: On the modularity of confluence of constructor-sharing term rewriting systems. In: CAAP 1994. LNCS, vol. 787, pp. 261-275 (1994)

[23] Sternagel, C., Middeldorp, A.: Root-labeling. In: RTA 2008. LNCS, vol. 5117, pp. 336-350 (2008)

[24] TeReSe: Term Rewriting Systems. vol. 55 of Cambridge Tracts in Theoretical Computer Science. Cambridge University Press (2003)

[25] Thiemann, R.: The DP Framework for Proving Termination of Term Rewriting. PhD thesis, RWTH Aachen (2007). Available as technical report AIB-2007-17

[26] Thiemann, R., Sternagel, C.: Certification of termination proofs using CeTA. In: Proc. of the 22nd International Conference on Theorem Proving in Higher Order Logics (TPHOLs 2009) 2009. LNCS, vol. 5674, pp. 452-468 (2009) 
[27] Waldmann, J.: Weighted automata for proving termination of string rewriting. J. Autom. Lang. Comb. 12(4), 545-570 (2007)

[28] Waldmann, J.: Polynomially bounded matrix interpretations. In: RTA 2010. LIPIcs, vol. 6, pp. 357-372 (2010)

[29] Zankl, H., Hirokawa, N., Middeldorp, A.: Uncurrying for innermost termination and derivational complexity. In: HOR 2011. EPTCS, vol. 49, pp. 46-57 (2011)

[30] Zankl, H., Korp, M.: The derivational complexity of the bits function and the derivation gap principle. In: WST 2010. (2010). 5 pages

[31] Zankl, H., Korp, M.: Modular complexity analysis via relative complexity. In: RTA 2010 . LIPIcs, vol. 6, pp. 385-400 (2010)

[32] Zankl, H., Korp, M.: On implementing modular complexity analysis. In: IWIL 2010. EPiC Series, vol. 2, pp. 42-47 (2010)

[33] Zankl, H., Middeldorp, A.: Satisfiability of non-linear (ir)rational arithmetic. In: LPAR 16. LNCS (LNAI), vol. 6355, pp. 481-500 (2010) 